Draft Version OCtober 3, 2018

Preprint typeset using $\mathrm{LAT}_{\mathrm{EX}}$ style emulateapj v. 26/01/00

\title{
COLD AND WARM ATOMIC GAS AROUND THE PERSEUS MOLECULAR CLOUD I: BASIC PROPERTIES
}

\author{
Snežana Stanimirović ${ }^{1}$, Claire Murray ${ }^{1},{\text { Min-Young } \text { LeE }^{2}, \text { Carl Heiles }^{3} \text {, Jesse Miller }}^{4,1}$ \\ Accepted for publication in ApJ
}

\begin{abstract}
Using the Arecibo Observatory we have obtained neutral hydrogen (HI) absorption and emission spectral pairs in the direction of 26 background radio continuum sources in the vicinity of the Perseus molecular cloud. Strong absorption lines were detected in all cases allowing us to estimate spin temperature $\left(T_{s}\right)$ and optical depth for 107 individual Gaussian components along these lines of sight. Basic properties of individual HI clouds (spin temperature, optical depth, and the column density of the cold and warm neutral medium, CNM and WNM) in and around Perseus are very similar to those found for random interstellar lines of sight sampled by the Millennium HI survey. This suggests that the neutral gas found in and around molecular clouds is not atypical. However, lines of sight in the vicinity of Perseus have on average a higher total HI column density and the CNM fraction, suggesting an enhanced amount of cold HI relative to an average interstellar field. Our estimated optical depth and spin temperature are in stark contrast with the recent attempt at using Planck data to estimate properties of the optically thick HI. Only $\sim 15 \%$ of lines of sight in our study have a column density weighted average spin temperature lower than $50 \mathrm{~K}$, in comparison with $\gtrsim 85 \%$ of Planck's sky coverage. The observed CNM fraction is inversely proportional to the optical-depth weighted average spin temperature, in excellent agreement with the recent numerical simulations by Kim et al. While the CNM fraction is on average higher around Perseus relative to a random interstellar field, it is generally low, $10-50 \%$. This suggests that extended WNM envelopes around molecular clouds, and/or significant mixing of CNM and WNM throughout molecular clouds, are present and should be considered in the models of molecule and star formation. Our detailed comparison of HI absorption with CO emission spectra shows that only $3 / 26$ directions are clear candidates for probing the CO-dark gas as they have $N(H I)>10^{21} \mathrm{~cm}^{-2}$ yet no detectable $\mathrm{CO}$ emission.
\end{abstract}

Subject headings: ISM: clouds — ISM: structure — radio lines: ISM

\section{INTRODUCTION}

Most of molecular gas in galaxies is assembled into giant molecular clouds (GMCs) with masses of $10^{4}-10^{7} \mathrm{M}_{\odot}$ (Fukui \& Kawamura 2010). Stars appear intimately associated with the dense regions of these GMCs (Lada et al. 2010), and recent observations suggest that the depletion timescale of molecular gas by star formation does not vary greatly across a wide range of galaxy environments (Schruba et al. 2011; Shetty et al. 2014). This strongly suggests that the ability to form molecular gas in the first place holds the key to understanding the evolutionary tracks of galaxies.

Atomic hydrogen has been considered for decades as the main formation reservoir of GMCs (Shu 1973; Blitz et al. 2007; Kim \& Ostriker 2006; Audit \& Hennebelle 2005; Heitsch et al. 2005; Clark et al. 2012). Although how exactly GMCs form out of the diffuse atomic medium is still not understood, the HI envelopes frequently observed around GMCs are likely to represent the material left over from the formation epoch and/or a product of photodissociation of molecular gas. In either case, these envelopes play a very important role in the GMC evolution and could explain long-standing questions such as the origin of the internal turbulent energy in GMCs.
Theoretical models considering the ongoing accretion of atomic material from the envelope onto GMCs are able to reproduce the level of observed turbulence, as well as the total GMC mass (Chieze \& Pineau Des Forets 1989; Hennebelle \& Inutsuka 2006; Goldbaum et al. 2011). In addition, it has been suggested that the GMC history is highly dependent on the initial surface density of the HI envelope. As shown by Goldbaum et al. (2011), only a factor of two increase of the HI surface density of the envelope from 8 to $16 \mathrm{M}_{\odot} \mathrm{pc}^{-2}$ is enough to decide whether or not a GMC mass will reach $\sim 10^{6} \mathrm{M}_{\odot}$ over a typical lifetime of 10-20 Myr.

While the HI envelopes around molecular clouds have been largely observationally studied via HI emission (Wannier et al. 1983, 1991; Andersson \& Wannier 1993; Fukui et al. 2009), traditionally HI has not been considered as very important for understanding molecule and star formation. For example, many GMC studies trying to estimate the $\mathrm{H}_{2}$ distribution from dust emission have neglected to account for HI as it was assumed that GMCs are highly dominated by molecular gas (e.g. Pineda et al. $(2008)$ ). In addition, a strong correlation between the star formation rate and the $\mathrm{H}_{2}$ surface density in galaxies has been considered as an evidence that only $\mathrm{H}_{2}$ is directly

\footnotetext{
${ }^{1}$ Department of Astronomy, University of Wisconsin, Madison, WI 53706; sstanimiastro.wisc.edu

${ }^{2}$ Laboratoire AIM, CEA/IRFU/Service d'Astrophysique, Bat 709, 91191 Gif-sur-Yvette, France

${ }^{3}$ Department of Astronomy, UC Berkeley, 601 Campbell Hall, Berkeley, CA 94720

${ }^{4}$ Department of Physics and Astronomy, Washington State University, PO Box 642814, Pullman WA 99164-2814
} 
related to star formation. However, recent extragalactic studies showed that globally across galaxies at kpc-scales, as well as in resolved studies at sub-kpc scales, the HI surface density $\Sigma_{\mathrm{HI}} \leqslant 10 \mathrm{M}_{\odot} \mathrm{pc}^{-2}$ (Wong \& Blitz 2002; Blitz \& Rosolowsky 2004; Bigiel et al. 2008; Schruba et al. 2011), re-opening interest in the role of $\mathrm{HI}$ shielding in molecule formation.

To investigate the formation of $\mathrm{H}_{2}$ from a theoretical point of view, and building up on several earlier studies (Spitzer \& Jenkins 1975; Elmegreen 1993), Krumholz et al. (2009) (KMT09) considered the structure of a photodissociation region (PDR) in a spherical cloud that is embedded in a uniform and isotropic radiation field. Their model is based on the balance between $\mathrm{H}_{2}$ formation on dust grains and photodissociation by Lyman-Werner (LW) photons and provides an analytic function for the $\mathrm{H}_{2}$ fraction as a function of the gas surface density. Their most important prediction is that a certain amount of the HI surface density, $\Sigma_{\mathrm{HI}}$, is required for shielding of $\mathrm{H}_{2}$ against photodissociation. Once this minimum $\Sigma_{\mathrm{HI}}$ is achieved, additional $\mathrm{HI}$ is fully converted into $\mathrm{H}_{2}$ and therefore $\Sigma_{\mathrm{HI}}$ saturates while $\Sigma_{\mathrm{H} 2}$ linearly increases. At solar metallicity, KMT09 predict $\Sigma_{\mathrm{HI}} \sim 10 \mathrm{M}_{\odot} \mathrm{pc}^{-2}$ as the minimum $\Sigma_{\mathrm{HI}}$ required for $\mathrm{H}_{2}$ formation, this is equivalent to the HI column density of $1.2 \times 10^{21} \mathrm{~cm}^{-2}$.

To investigate the role of $\mathrm{HI}$ shielding on sub-pc scales in Lee et al. (2012) we mapped the transition from Hi to $\mathrm{H}_{2}$ across the Perseus molecular cloud 5 . The HI data in this study were from the GALFA-Hi survey (Peek et al. 2011; Stanimirović et al. 2006) and the HI column density was estimated under the optically thin assumption. To estimate the $\mathrm{H}_{2}$ image, the 60 and $100 \mu \mathrm{m}$ data from the Improved Reprocessing of the IRAS Survey (IRIS) (Miville-Deschênes \& Lagache 2005) were used. We derived $T_{\text {dust }}$ from the $I_{60} / I_{100}$ ratio, and then converted $\tau_{100}$ to $A_{V}$ by finding a proportionality constant between our derived $A_{V}$ and the $A_{V}$ image derived from optical extinction (provided by the COMPLETE survey, Ridge et al. (2006)). Finally, the $\mathrm{H}_{2}$ column density was calculated as: $N\left(\mathrm{H}_{2}\right)=\left(A_{V} / \mathrm{DGR}-N(\mathrm{HI})\right) / 2$; the dust-to-gas ratio DGR $=1.1 \times 10^{-21} \mathrm{mag} \mathrm{cm}^{2}$ was measured locally around Perseus.

The key result from Lee et al. (2012) is the detection of an almost constant $\Sigma_{\mathrm{HI}}$ of $6-8 \mathrm{M}_{\odot} \mathrm{pc}^{-2}$ for several dark and star-forming regions in Perseus. This is in agreement with KMT09's prediction for the saturation of $\Sigma_{\mathrm{HI}}$. In addition, Lee et al. showed that $\mathrm{H}_{2}$ extends up to $20 \mathrm{pc}$ from core centers, and that the HI envelope is very extended (>20 pc). The HI halo of Perseus was previously studied by Andersson \& Wannier (1993) who focused on dark region B5. Using radiative transfer modeling they found that the HI halo is about $5 \times 8$ pc in size.

While the observed flattening of $\Sigma_{\mathrm{HI}}$ can be attributed to the conversion of $\mathrm{HI}$ into $\mathrm{H}_{2}$ as in KMT09, an alternative possibility is that $\Sigma_{\mathrm{HI}}$ is simply underestimated due to the presence of high optical depth HI which is not fully measured in emission line observations. The high optical depth $\mathrm{HI}$ can be measured from self-absorption features, caused by the background Galactic HI emission being absorbed by the cooler foreground HI (Knapp 1974; Goodman \& Heiles 1994; Li \& Goldsmith 2003). Many narrow self-absorption features have been considered as kinematically associated with CO and have inferred temperature of less than $40 \mathrm{~K}$ and the atomic hydrogen column density fraction of only 0.0016 relative to $\mathrm{H}_{2}$. If $\mathrm{HI}$ is a dissociation product of $\mathrm{H}_{2}$, these measurements suggest a cloud age of 3-30 Myrs Goldsmith \& Li (2005). While self-absorption can provide spatial information about the cold HI, e.g. Gibson et al. (2000), it always requires complicated line modeling and is limited by the ability to clearly distinguish self-absorption features from temperature fluctuations and/or multiple individual line of sight components.

The main aim of this study is to investigate the effect of high optical depth on the HI surface density saturation observed in Lee at al. (2012). We use the most direct way to estimate the "true" HI column density by measuring HI absorption against radio continuum sources located behind Perseus. We use these observations to investigate properties of the cold and warm HI around Perseus (Paper I), as well as to derive the ratio of the true HI column density to the HI column density derived under the optically thin assumption (Paper II).

The structure of this study is organized in the following way. In this paper (Paper I) we focus on the properties of cold gas around Perseus. Our observing and data processing strategies are explained in Section 2, and in Section 3 we summarize the methodology used to estimate spin temperature and column density of the cold neutral medium (CNM) and the warm neutral medium (WNM). In Section 4 we investigate the basic physical properties of atomic gas in the Perseus HI envelope, and in Section 5 we compare HI absorption and carbon monoxide (CO) emission spectra. We summarize our results in Section 6. In Paper II we estimate the correction for high optical depth using our HI absorption measurements, apply this correction and re-visit the question of HI saturation in Perseus.

\section{OBSERVATIONS AND DATA REDUCTION}

\subsection{HI absorption observations}

We selected 27 radio continuum sources from the NVSS survey (Condon et al. 1998), located over an area of roughly 500 square degrees centered on Perseus with flux densities at $1.4 \mathrm{GHz}$ greater than $0.8 \mathrm{Jy}$. Figure 1 shows the source positions overlaid on the $\mathrm{H}_{2}$ surface density image of Perseus from Lee et al. (2012). Source information (RA, Dec, flux density at $21 \mathrm{~cm}$, and the diffuse background radio continuum emission) is given in Table 1.

The observations were conducted with the Arecibo telescope6. Using the L-wide receiver, we simultaneously recorded spectra centered at $1420 \mathrm{MHz}$ and the two $\mathrm{OH}$ main lines (1665 and $1667 \mathrm{MHz}$ ), achieving a velocity resolution of $0.16 \mathrm{~km} \mathrm{~s}^{-1}$. We sampled simultaneously two linearly polarized channels performing both auto and cross-correlations with the Arecibo's three-level "interim" digital correlator. The Arecibo telescope has an angular resolution of $3.5^{\prime}$ at these frequencies. As shown by

\footnotetext{
${ }^{5}$ Perseus is located at a distance of 200-350 pc (Herbig \& Jones 1983), has $M \sim 2 \times 10^{4} \mathrm{M}_{\odot}$ (Sancisi et al. 1974; Lada et al. 2010) and solar metallicity (González Hernández et al. 2009).

6 The Arecibo Observatory is operated by SRI International under a cooperative agreement with the National Science Foundation (AST1100968), and in alliance with Ana G. Méndez-Universidad Metropolitana, and the Universities Space Research Association.
} 
TABLE 1

SourCE LIST

\begin{tabular}{ccccc}
\hline \hline Source & $\begin{array}{c}\text { RA (J2000) } \\
(\mathrm{h} \mathrm{m} \mathrm{s})\end{array}$ & $\begin{array}{c}\text { Dec }(\mathrm{J} 2000) \\
\left({ }^{\circ}{ }^{\prime \prime}\right)\end{array}$ & $\begin{array}{c}T_{\text {src }} \\
(\mathrm{Jy})\end{array}$ & $\begin{array}{c}T_{\text {sky }} \\
(\mathrm{K})\end{array}$ \\
\hline $\mathrm{NV0157+28}$ & $01: 57: 12.85$ & $28: 51: 38.49$ & 1.4 & 2.782 \\
4C+29.05 & $02: 01: 35.91$ & $29: 33: 44.18$ & 1.2 & 2.785 \\
4C+27.07 & $02: 17: 01.89$ & $28: 04: 59.12$ & 1.0 & 2.785 \\
5C06.237 & $02: 20: 48.06$ & $32: 41: 06.64$ & 0.9 & 2.787 \\
B20218+35 & $02: 21: 05.48$ & $35: 56: 13.91$ & 1.7 & 2.790 \\
3C067 & $02: 24: 12.31$ & $27: 50: 11.69$ & 3.0 & 2.786 \\
4C+34.07 & $02: 26: 10.34$ & $34: 21: 30.45$ & 2.9 & 2.791 \\
NV0232+34 & $02: 32: 28.72$ & $34: 24: 06.08$ & 2.6 & 2.791 \\
3C068.2 & $02: 34: 23.87$ & $31: 34: 17.62$ & 1.0 & 2.787 \\
4C+28.06 & $02: 35: 35.41$ & $29: 08: 57.73$ & 1.3 & 2.788 \\
4C+28.07 & $02: 37: 52.42$ & $28: 48: 09.16$ & 2.2 & 2.790 \\
4C+34.09 & $03: 01: 42.38$ & $35: 12: 20.84$ & 1.9 & 2.794 \\
4C+30.04 & $03: 11: 35.19$ & $30: 43: 20.62$ & 1.0 & 2.792 \\
B20326+27 & $03: 29: 57.69$ & $27: 56: 15.64$ & 1.3 & 2.787 \\
4C+32.14 & $03: 36: 30.12$ & $32: 18: 29.47$ & 2.7 & 2.793 \\
3C092 & $03: 40: 08.55$ & $32: 09: 02.32$ & 1.6 & 2.791 \\
3C093.1 & $03: 48: 46.93$ & $33: 53: 15.41$ & 2.4 & 2.795 \\
4C+26.12 & $03: 52: 04.36$ & $26: 24: 18.11$ & 1.4 & 2.783 \\
B20400+25 & $04: 03: 05.61$ & $26: 00: 01.61$ & 0.9 & 2.785 \\
3C108 & $04: 12: 43.69$ & $23: 05: 05.53$ & 1.5 & 2.788 \\
B20411+34 & $04: 14: 37.28$ & $34: 18: 51.31$ & 1.9 & 2.793 \\
4C+25.14 & $04: 20: 49.30$ & $25: 26: 27.63$ & 1.0 & 2.785 \\
4C+33.10 & $04: 47: 08.90$ & $33: 27: 46.85$ & 1.2 & 2.799 \\
3C131 & $04: 53: 23.35$ & $31: 29: 25.36$ & 2.9 & 2.801 \\
3C132 & $04: 56: 43.08$ & $22: 49: 22.27$ & 3.4 & 2.795 \\
4C+27.14 & $04: 59: 56.10$ & $27: 06: 02.19$ & 0.9 & 2.796 \\
3C133 & $05: 02: 58.51$ & $25: 16: 25.16$ & 5.8 & 2.796 \\
\hline & & & &
\end{tabular}


Heiles \& Troland (2003a) in their Millennium HI survey, Arecibo can accurately measure HI absorption lines for strong sources (flux density larger than $\sim 1 \mathrm{Jy}$ ).

The observing procedure used was the same as in Heiles \& Troland (2003a) and Stanimirović \& Heiles (2005). This technique employs a 17 -point observing pattern including 16 off-source measurements and one onsource measurement. The pattern was designed to measure the first and second derivatives of the 21-cm intensity fluctuations on the sky, and also to fine-tune for the instrumental effects involving the system gain. The autocorrelation data were used to derive the "expected" HI emission profile $\left(T_{\exp }\right)$, which is the profile that would be observed at the source position if the continuum sources were absent, the optical depth profile $(\tau)$, and their uncertainties. With 17 measurements, the off-source spectra are expressed in a Taylor series expansion of the expected profile and a small contribution from the source intensity attenuated by the optical depth. A least-squares fitting technique is then used to estimate the optical depth profile, the expected profile and its spatial derivatives, and the off-source gain simultaneously (Heiles \& Troland 2003a). However, our updated data reduction software takes a slightly simpler approach by not including the fine-tuning of gain variations under the assumption that the on-axis telescope gain and the beam properties vary spatially and a detailed knowledge of these variations is required to estimate properly off-axis gains. Therefore, we just derive the optical depth profile, the expected profile and its spatial derivatives for each of 16 off positions. These are used to derive the uncertainty spectra for both the expected emission and optical depth spectra.

We have experimented with using the first order Taylor expansion instead of the second order. For all sources we find that the difference between optical depth profiles derived used the two expansions is within 1- $\sigma$ uncertainty. While the second order expansion is clearly more accurate (has smaller systematic errors), the derived $T_{\exp }(v)$ and $\tau(v)$ are noiser than when using the first-order expansion. The increased noise comes from fitting a larger number of unknown parameters, and also from a large covariance between the second derivatives of the expected profile, $T_{\exp }(v)$, and $\tau(v)$. We tolerate the slightly higher noise for better accuracy of derived profiles and therefore use the second-order Taylor expansion for all sources.

Following the data reduction, for all sources we obtained an HI absorption spectrum $\left(e^{-\tau(v)}\right)$, an HI (expected) emission spectrum $\left(T_{\exp }(v)\right)$, and their uncertainty profiles. A main beam efficiency of $\eta=0.85$ (based on calibration measurements at Arecibo, Perillat et al.) was used to convert $T_{\exp }(v)$ from the antenna temperature units to the brightness temperature scale. With an integration time on average of about 1 hour, we achieved an rms noise level in optical depth of $\sim 1 \times 10^{-3}$ per $1 \mathrm{~km} \mathrm{~s}^{-1}$ velocity channel.

Inspection of derived profiles revealed that several sources have small positive spectral features in their optical depth profiles at a level slightly higher than the 1- $\sigma$ uncertainty and highly localized in velocity. This effect is a result of high spatial derivatives of the HI emission (due to the presence of significant small-scale structure) and suggests that even the second-order Taylor expansion is not a good representation of the measured off positions in several cases. These sources are: $4 \mathrm{C}+27.14$ and $4 \mathrm{C}+33.10$. In addition, $4 \mathrm{C}+33.10$ has very broad both absorption and emission profiles with many velocity components and its component fitting is more difficult and ambiguous than for other sources. However, in order to use as many sources as possible and considering that small artifacts are very localized in velocity, we include these three sources in our analysis (but make sure that artifacts are not fitted as real features). One source that we exclude from analysis is $4 \mathrm{C}+32.14$ which has a highly saturated absorption profile and therefore all fitted parameters are highly uncertain for this source.

\subsubsection{Comparison with HT03}

Several of our sources were observed previously by Heiles \& Troland (2003b) (from now on HT03): 3C+93.1, 3C131, 3C132, and 3C133. In terms of optical depth spectra, our results for $3 \mathrm{C}+93.1,3 \mathrm{C} 132$, and $3 \mathrm{C} 133$ agree extremely well with $\mathrm{HT} 03$, within $3 \%$. In the case of $3 \mathrm{C} 131$ we find a slightly larger difference, but this is still within the 3- $\sigma$ uncertainty. In case of expected profiles expressed in terms of antenna temperature, for all sources we find excellent agreement with HT03. We do correct our expected profiles for the beam efficiency and work with brightness temperature profiles in this paper.

\subsection{HI emission data from the GALFA-HI survey}

To investigate different methods for the derivation of the correction for high optical depth (focus of Paper II), as well as to estimate the importance of stray radiation, we also use the $\mathrm{HI}$ emission data from the Galactic Arecibo L-band Feed Array Survey in HI (GALFA-HI). GALFAHI uses ALFA, a seven-beam array of receivers mounted at the focal plane of the 305-m Arecibo telescope, to map Hi emission in the Galaxy. Each of seven dual polarization beams has an effective beamsize of $3.9^{\prime} \times 4.1^{\prime}$ and a gain of 8.5-11 Jy K ${ }^{-1}$ (Peek et al. 2011). The GALFA-Hi spectrometer, GALSPECT, has a velocity resolution of 0.184 $\mathrm{km} \mathrm{s}^{-1}(872 \mathrm{~Hz})$ and covers $-700 \mathrm{~km} \mathrm{~s}^{-1}<v<+700 \mathrm{~km}$ $\mathrm{s}^{-1}(7 \mathrm{MHz})$ in the Local Standard of Rest (LSR) frame7.

In Lee et al. (2012) we combined scans from several GALFA-HI projects and produced an Hi cube of Perseus centered at $(\mathrm{RA}, \mathrm{Dec})=\left(03^{\mathrm{h}} 29^{\mathrm{m}} 52^{\mathrm{s}},+30^{\circ} 34^{\prime} 1^{\prime \prime}\right)$ in $\mathrm{J} 2000$ \& with a size of $14.8^{\circ} \times 9.0^{\circ}$. We use the same data here, but extend the data cube beyond Perseus to include locations of all radio continuum sources. This data cube has a size close to $60^{\circ} \times 18^{\circ}$, with a pixel size of $1^{\prime}$. After smoothing the cube to $36^{\prime}$ and comparing the average HI spectrum with the corresponding spectrum from the Leiden/Argentine/Bonn (LAB) survey (Kalberla et al. 2005), we derived the correction factor of 1.1 that needed to be applied on the pixel-by-pixel basis to fine-tune GALFAHI's calibration (we note that our data came from an early data reduction scheme, before the public GALFA-HI data cubes were finalized and released).

\footnotetext{
${ }^{7}$ All velocities quoted in this paper are in the kinematic or standard LSR frame, defined based on the average velocity of stars in the Solar neighborhood as: $20.0 \mathrm{~km} \mathrm{~s}^{-1}$ toward $\mathrm{RA}=18.0 \mathrm{hr}$, Dec $=30.0$ degrees in the 1900 epoch.

${ }^{8}$ All quoted coordinates in this paper are in J2000.
} 


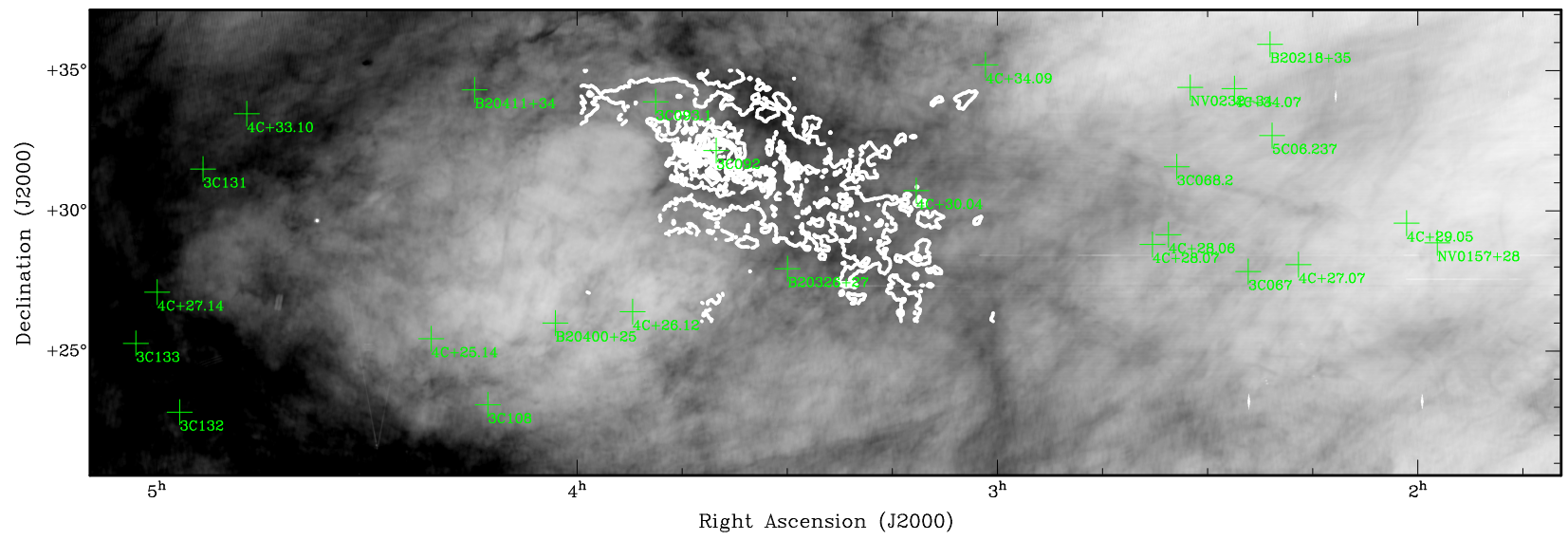

FIG. 1. - Positions of background radio continuum sources overlaid on the HI column density produced using GALFA-HI data at angular resolution of $\sim 4^{\prime}$. The intensity scale ranges from $2.2 \times 10^{19} \mathrm{~cm}^{-2}$ to $1.5 \times 10^{21} \mathrm{~cm}^{-2}$. White contours show the $\mathrm{H}_{2}$ surface density distribution of Perseus from Lee et al. (2012). Contour levels range from 5 to $90 \%$ of the peak value $\left(4.6 \times 10^{21} \mathrm{~cm}^{-2}\right)$, with a step of $10 \%$.

Lee et al. (2012) also used the GALFA-HI data to investigate the $\mathrm{HI}$ saturation in Perseus. To estimate the HI column density, the HI emission was integrated from $v_{\mathrm{LSR}}=-5$ to $15 \mathrm{~km} \mathrm{~s}^{-1}$. This range was selected as resulting in the maximum correlation between $N(\mathrm{HI})$ and the $A_{V}$ image from 2MASS (Ridge et al. 2006), exploring the idea that in mainly diffuse, low- $\mathrm{A}_{V}$ regions of Perseus where molecular gas is not abundant $\mathrm{HI}$ correlates well with $\mathrm{A}_{V}$.

\subsection{Stray radiation consideration for HI emission}

Both our derived expected HI emission profiles and HI spectra from the GALFA-HI survey may be affected by stray radiation. Stray radiation is caused by radiation entering through higher order sidelobes and can result in broad, weak emission features. Correcting for stray radiation is a complex problem and requires a detailed knowledge of the Arecibo telescope beam and how it varies with azimuth and elevation. In this paper we provide only a rough check of our spectra relative to the LAB survey, which has been meticulously corrected for stray radiation. We take a twofold approach: (i) we compare our derived expected profiles $T_{\text {exp }}$ with the HI spectra from the GALFA-HI survey and find good agreement (within our estimated uncertainties); (ii) we then smooth the GALFA$\mathrm{HI}$ data cube to the same angular and velocity resolution of the LAB survey $\left(36^{\prime}\right)$, extract spectra at the positions of our continuum sources and compare them to search for broad wing-like features. We find that, in the majority of cases, the differences lie below the 1- $\sigma$ uncertainty level for our derived expected profiles. Therefore, we conclude that stray radiation is not a significant problem for this study. Our future work will develop a methodology for a detailed stray radiation correction.

\subsection{Additional data sets}

We use the CO (1-0) emission data from Dame et al. (2001) obtained with the $1.2 \mathrm{~m}$ telescope at the Harvard Smithsonian Center for Astrophysics (CfA) and at 8.4' angular resolution. We also use the integrated $\mathrm{CO}$ intensity $\left(W_{C O}\right)$ and $E(B-V)$ images from Planck (Planck Collaboration et al. 2013) with angular resolution of $5^{\prime}$. When using Planck data for comparison with Dame et al. (2001) we first smooth the Planck images to angular resolution of $8^{\prime}$ and regrid to make sure pixels are independent.

\section{ANALYSIS: COMPONENT FITTING OF HI ABSORPTION/EMISSION PAIRS}

To analyze HI absorption spectra we performed a decomposition into individual velocity components by employing the technique of Heiles \& Troland (2003a). This allows us to estimate spin temperature and the HI column density for individual CNM components. This technique assumes that the CNM contributes to both HI absorption and emission spectra, while the warm neutral medium (WNM) contributes only to the HI emission spectrum. The technique is based on the Gaussian decomposition of both absorption and emission spectra, and it takes into account the fact that a certain fraction of the WNM gas may be located in front of the CNM clouds, resulting in a portion of the WNM being absorbed by the CNM. All 
S. Stanimirović et al.
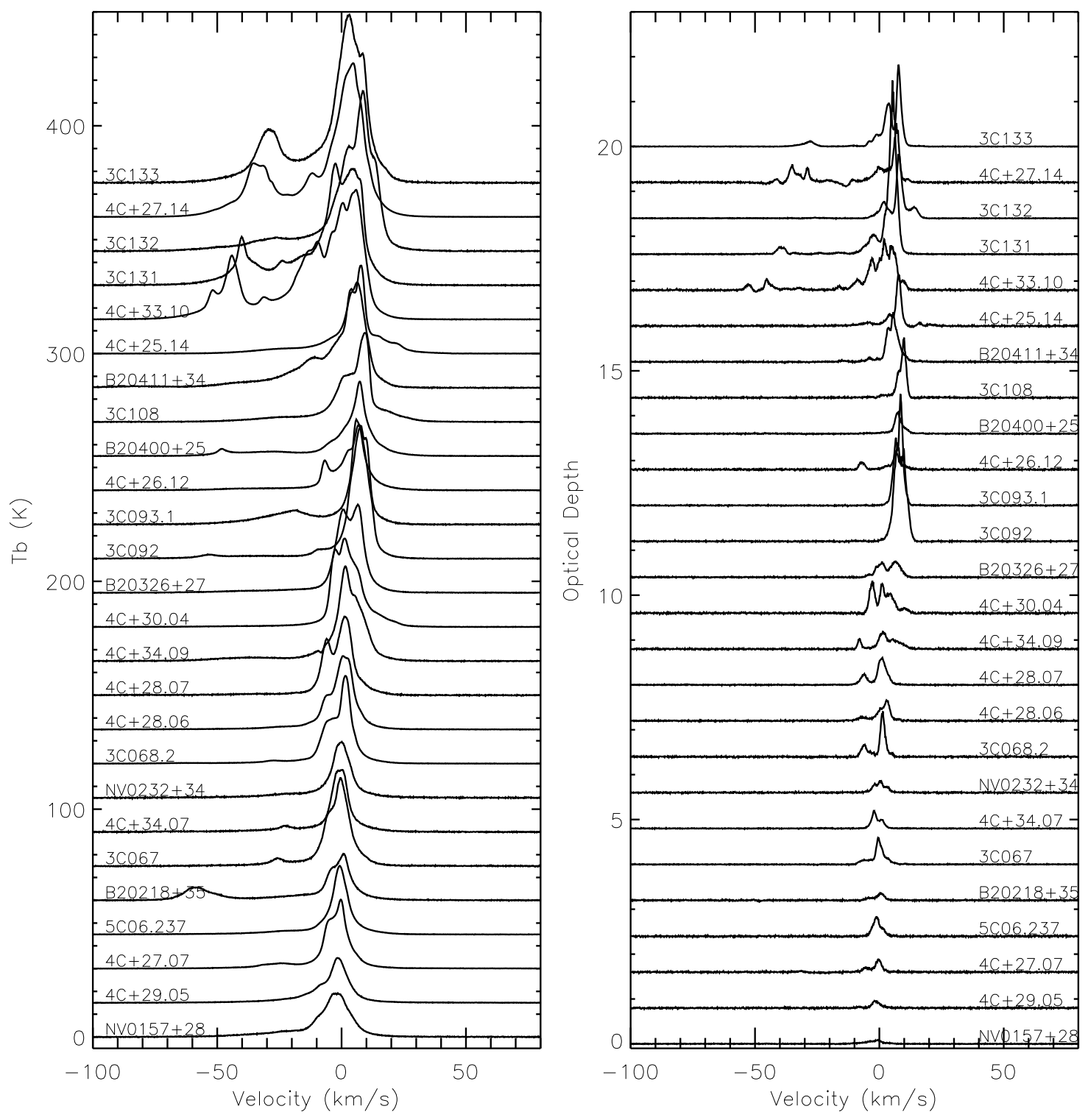

FIG. 2.- (left) Brightness temperature of expected profiles of all sources offset in y-axis by $15 \mathrm{~K}$ for comparison. (right) Optical depth profiles of all sources offset in y-axis by 0.8 for easy comparison. 
possible permutations of the CNM components along the line-of-sight have been taken into account when searching for the best fit. Pros and cons regarding the use of Gaussian functions to represent the CNM absorption profiles have been discussed in Heiles \& Troland (2003a).

We first fit $\tau(v)$ with a set of $N$ Gaussian functions using a least-squares technique:

$$
\tau(v)=\sum_{0}^{N-1} \tau_{0, n} e^{-\left[\left(v-v_{0, n}\right) / \delta v_{n}\right]^{2}}
$$

where $\tau_{0, n}$ is the peak optical depth, $v_{0, n}$ is the central velocity, and $\delta v_{n}$ is the $1 / \mathrm{e}$ width of component $n . N$ is the minimum number of components necessary to make the residuals of the fit smaller or comparable to the estimated noise level of $\tau(v)$.

While the optical depth spectrum predominantly reflects the CNM, both the cold and warm neutral media contribute to the expected HI emission spectrum:

$$
T_{\exp }(v)=T_{B, C N M}(v)+T_{B, W N M}(v) .
$$

The first term, $T_{B, C N M}(v)$, the $\mathrm{HI}$ emission originating from $N$ CNM components is:

$$
T_{B, C N M}(v)=\sum_{0}^{N-1} T_{s, n}\left(1-e^{-\tau_{n}(v)}\right) e^{-\sum_{0}^{M-1} \tau_{m}(v)},
$$

where $T_{s, n}$ is the spin temperature of cloud $n$, and the subscript $m$ represents each one of the $M$ CNM clouds that lie in front of cloud $n$.

Next, $T_{B, W N M}(v)$, the HI emission originating from the WNM, is represented with a set of $K$ Gaussian functions. The complicating factor here is that a certain fraction $F$ of the WNM is located in front of the CNM, while a fraction $(1-F)$ of the WNM is beyond the CNM with its emissions being absorbed by CNM clouds:

$T_{B, W N M}(v)=\sum_{0}^{K-1}\left[F_{k}+\left(1-F_{k}\right) e^{-\tau(v)}\right] \times T_{0, k} e^{-\left[\left(v-v_{0, k}\right) / \delta v_{k}\right]^{2}}$,

where the subscript $k$ corresponds to each of the WNM components and a fraction $F_{k}$ of the WNM cloud $k$ lies in front of all CNM components, while a fraction $1-F_{k}$ is being absorbed by the CNM clouds. To fit the corresponding emission spectra, we assume that the center and width of the absorption-selected CNM components are fixed and include a minimum number of additional WNM components to reduce the fit residuals to within the neighborhood of the $1-\sigma$ uncertainties. We use a certain number of WNM components and fit the $T_{\exp }(v)$ profile simultaneously for the Gaussian parameters of the WNM components and the spin temperature of individual CNM clouds, while assuming a given order of CNM clouds along the line of sight and a given set of $F_{k}$ values. We try to use the minimum number of WNM components such that the residuals of this fitting process are reasonably close to the $1-\sigma$ uncertainty for $T_{\text {exp }}$.

Please note that the expected profile in the left-hand side of equation (2) has been baseline corrected, which means that we measure $T_{\exp }(v)-T_{s k y}$, where $T_{s k y}$ contains contributions from the Cosmic Microwave Background (CMB) and the Galactic synchrotron emission. Before doing the radiative transfer calculations we estimate $T_{s k y}$ and add it back to the left-hand side of equation (2) by assuming $2.725 \mathrm{~K}$ for the CMB. To estimate the contribution from the Galactic synchrotron emission we use the Haslam et al. (1982) $408 \mathrm{MHz}$ survey of the Galaxy. The brightness temperature at $408 \mathrm{MHz}$ is converted to $1.4 \mathrm{GHz}$ using the spectral index of -2.7 . As the Galactic latitude of observed sources in the vicinity of Perseus is generally $>10$ degrees, the synchrotron contribution is small and $T_{s k y}$ ranges from 2.78 to $2.80 \mathrm{~K}$ in our case (Table 1).

For each source, we vary the order of Gaussian functions along the line-of-sight (for $N$ CNM components there are $N$ ! possible orderings) and perform the $T_{\exp }(v)$ fit. We then choose the ordering of CNM components that gives the smallest residuals in the least-squares fit. Unfortunately, the difference in the fit residuals is often not sufficiently statistically significant to distinguish between different values of $F_{k}$. However, $F_{k}$ has a large effect on the derived spin temperatures. Hence we follow the Heiles \& Troland (2003a) suggestion and estimate the final spin temperatures by assigning characteristic values of $0,0.5$, or 1 to each $F_{k}$ (among the extreme possible values of 0 and 1 ), and repeating this for all possible combinations of WNM clouds. The final spin temperatures are then derived as a weighted average over all trials.

Out of 26 sources, 23 have well-constrained fits. Three sources, 3C133, 3C131 and 4C+25.14, have more than 6 individual CNM components in their absorption spectra. The corresponding fit for the spin temperature of these components in the presence of WNM features in emission is therefore more complicated, and the fitting process does not converge. Furthermore, for 6 sources (3C068.2, 3C133, $4 \mathrm{C}+25.14,4 \mathrm{C}+28.07,4 \mathrm{C}+30.04$, and $\mathrm{B} 20411+34)$ the fitted height of one absorption component is too small to be reliably recovered in the corresponding emission spectrum. Thus, the spin temperatures for these 6 components are calculated to be less than $1 \mathrm{~K}$. Increasing the spin temperature by hand does not significantly degrade the quality of the fit. Therefore, for these uncertain components, we set the spin temperature equal to the uncertainty in $T_{s}$ derived from the iterations over CNM component orders along the line of sight and fraction of WNM absorbed. The error on this value is set to the median $T_{s}$ error for components along all 26 lines of sight, or $6.75 \mathrm{~K}$.

\section{PROPERTIES OF COLD AND WARM GAS AROUND PERSEUS}

Figure 2 shows emission and absorption spectra for all sources except $4 \mathrm{C}+32.14$ which has a saturated optical depth profile. Strong absorption lines were detected in the direction of all sources. In all cases, the strongest emission and absorption is found at $\sim 0 \mathrm{~km} \mathrm{~s}^{-1}$, and is generally well confined within the range of -20 to $20 \mathrm{~km} \mathrm{~s}^{-1}$ (see also Figure 6$)$. However, in the case of four sources $(4 \mathrm{C}+33.10$, $4 \mathrm{C}+27.14,3 \mathrm{C} 133$, and $3 \mathrm{C} 131)$ there are strong emission and absorption features around $-40 \mathrm{~km} \mathrm{~s}^{-1}$. Visual inspection of velocity components close to Perseus using the GALFA-HI data cubes suggests that this secondary region is likely not associated with Perseus.

We show results of our Gaussian component fitting for 4 example sources in Figures 3 and 4 . In each panel of both figures we plot the derived expected emission and optical depth profiles for an individual source. For the optical depth spectra, we overplot the individual CNM compo- 
nents (dotted lines), as well as the residuals for the fit (offset to the bottom of the panel) with the derived uncertainties for the spectrum for comparison. For the expected emission profiles, we overplot the sum of all WNM components (dot-dashed line), the total $T_{s}$-corrected contribution of the CNM (thick dashed line), and the fit residuals (shown below zero in the panel) with the uncertainties in the profile for comparison. The two sources in Figure 3 . $3 \mathrm{C} 131$ and $4 \mathrm{C}+27.14$, have broad HI profiles as likely include emission/absorption beyond Perseus, 3C092 in Figure 4 is located behind the main body of Perseus, and NVO0157+26 in the same figure is an example of a low optical depth profile.

In Table 2, we list the Gaussian parameters associated with all CNM and WNM components for each source. In column 1 we list the peak brightness temperature for each component. For the WNM components, this is equal to the unabsorbed Gaussian height and estimated error in the fit. For the CNM components, this is equal to the calculated spin temperature multiplied by $\left(1-e^{-\tau}\right)$, as in Equation 3 , and is quoted without uncertainty. In columns 2 and 3 we list the centers and FWHM of CNM and WNM components with estimated fit uncertainties. In column 4, we list the peak optical depth of each component. For the CNM components, this is equal to the height of each component

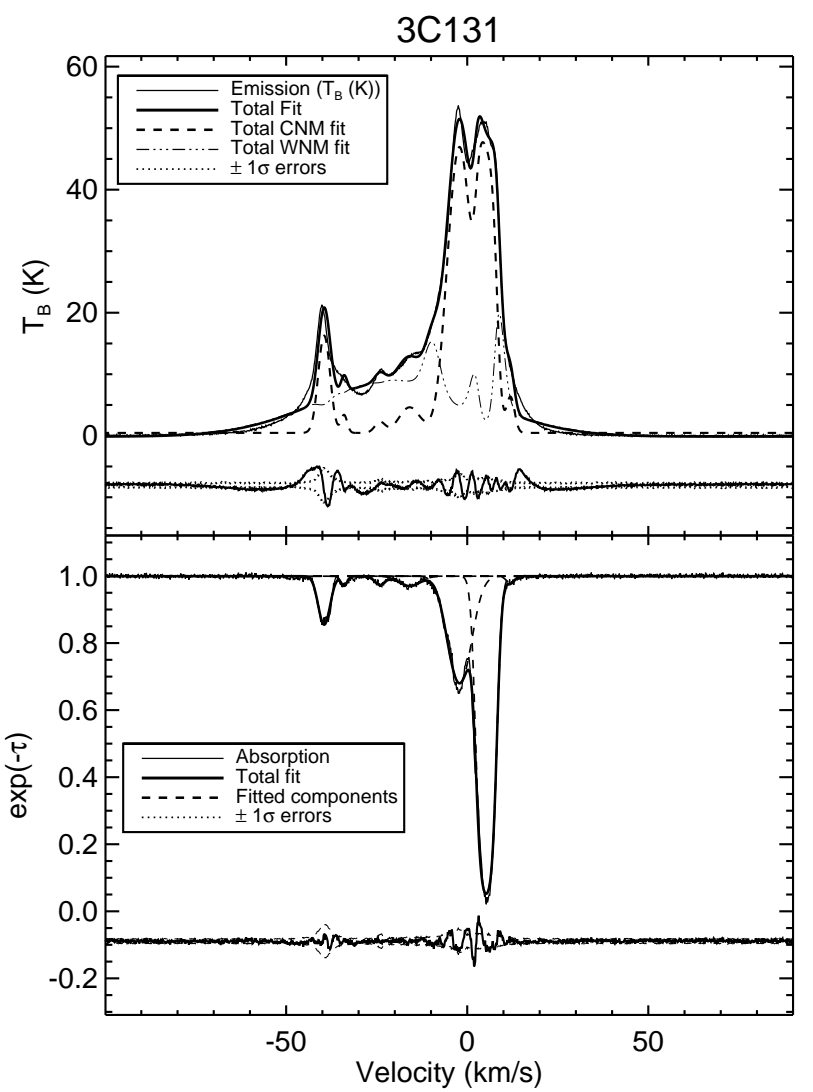

(in $\tau$ ), with associated uncertainty. For the WNM components, this is equal to the maximum contribution of each WNM component detected in emission to the absorption profile, and is found by measuring the height of the absorption fit residuals at the central velocity of each WNM component. In column 5 we list the spin temperatures, which for the CNM components is equal to the calculated values from the fit with fit uncertainties. For the WNM components, this is equal to a lower limit imposed by the upper limit on optical depth in column 4 , and these values are also quoted without error because the errors are extremely large due to the nature of the estimation process. In column 6 , we list the maximum kinetic temperature of each component based on the line widths. In column 7 , we list the HI column density of each individual component, and these values are quoted in units of $10^{20} \mathrm{~cm}^{-2}$. Finally, in column 8 we list the fraction of each WNM component lying in front of all CNM components $(F$, either $0.0,0.5$ or 1.0, see Section 2.2) or the order of each CNM component along the line of sight ( $O$, integer values).

\subsection{Optical Depth}

A summary of the fitting results is presented in Figures 5 to 8 As shown in Figure 5 (a), the median peak optical depth $\tau_{\max }$ for individual Gaussian components is 0.16, and only a handful of CNM components has $\tau_{\max }>1$

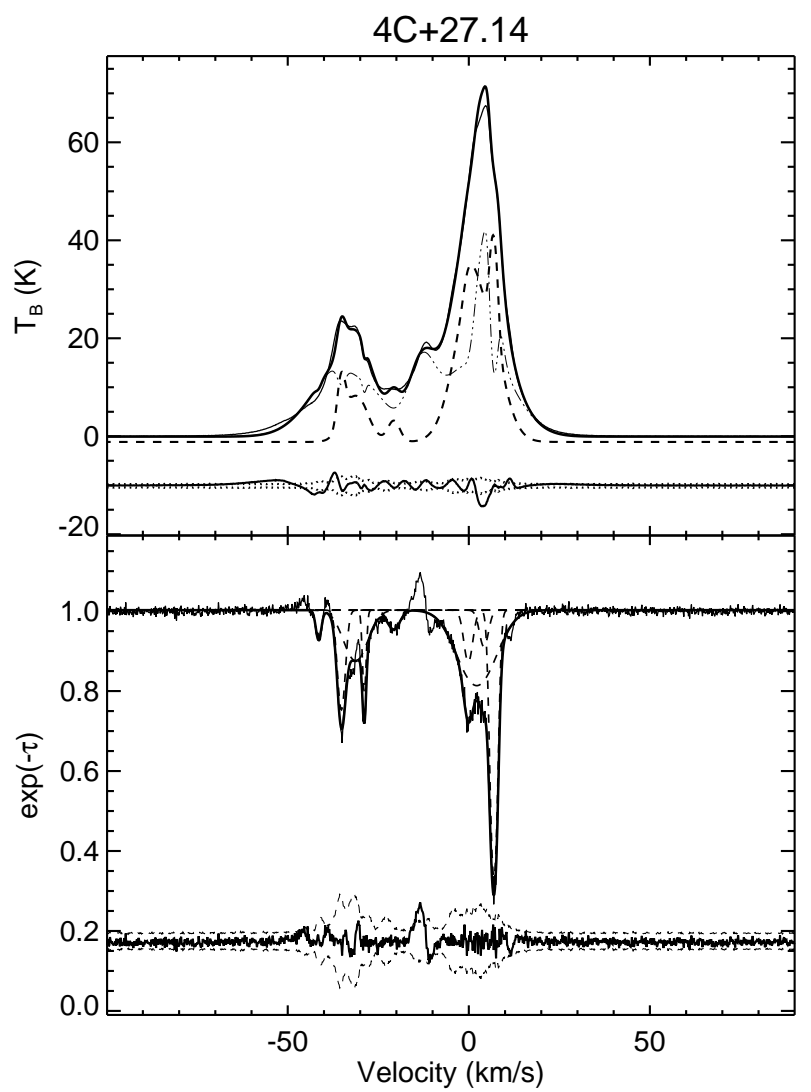

Fig. 3.- Example Gaussian fits to emission and absorption spectra. (Left) 3C131, (Right) 4C+27.14. In the top panels, the thin solid line is the expected profile, $T_{\exp }$ (see Section 2.1 for derivation). The thin dot-dashed lines display the sum of WNM Gaussian components and the thick dashed lines display the total contribution to the $T_{\exp }$ profile by the CNM from the absorption profile. The thick solid line is the total WNM and CNM fit. The residuals from the fit are plotted below zero, with $+/-\Delta T_{\text {exp }}$ overplotted. In the bottom panel, the thin solid line shows the optical depth profile $\left(e^{-\tau}\right)$, with CNM components displayed in the thin dotted lines and the thick solid line representing the fit to the optical depth profile. The residuals from the fit are plotted at the bottom of the figure, with $+/-\Delta e^{-\tau}$ overplotted. 
(10/107). Perseus is an intermediate-mass GMC located about 20 degrees below the Galactic plane and may not sample the densest molecular gas. In addition, a tighter grid of background sources may be able to sample better denser gas. Only two of our sources are located right behind the main body of Perseus. Their peak optical depth is 1.5 .

The same figure shows $\tau_{\max }$ for the components from HT03, dotted lines show median rms noise in optical depth for two studies. The two studies agree very well and have relatively similar (median) sensitivity, but we are missing the low- $\tau_{\max }$ portion of the distribution. This could be partially due to our small survey area relative to HT03 who had more sources at high Galactic latitudes. We note that HT03's sensitivity varies across sources as their survey was searching for strong sources suitable for Zeeman measurements.

Very recently, Fukui et al. (2014b) suggested a new approach to estimate properties (optical depth and spin temperature) of cold HI by utilizing dust emission. They noticed that the Planck dust optical depth $\tau_{353}$ at $353 \mu \mathrm{m}$ correlates with $N(H I)$, but the scatter in this relation is much smaller when different dust temperature regimes are considered separately. By assuming that the highest dust temperature sub-sample is associated with the opticallythin $\mathrm{HI}$, the saturation seen in the $\tau_{353}-N(H I)$ relation was attributed to the existence of the high optical depth HI solely. By inverting the relation, they estimated a single value of $T_{s}$ and $\tau_{H I}$ per pixel from their all-sky $\tau_{353}$ images (after masking low-latitude regions with $|b|<15$ degrees and regions with internal dust heating as traced by the $\mathrm{H} \alpha$ emission). They found that $85 \%$ of data points have $\tau_{H I}>0.5$ and $T_{s}<40 \mathrm{~K}$. Similar results were obtained for the high latitude clouds MBM 53-55 (Fukui et al. 2014a), increasing the HI mass of MBM 53-55 clouds by a factor of two. Fukui et al. (2014b) suggested that the local interstellar medium (ISM) may be dominated by the high optical depth HI, and that this component may explain all of the CO-dark gas in the Milky Way.

Around Perseus we find $\tau_{\max }>0.5$ only for 21 out of 107 (20\%) individual (Gaussian) components. This is clearly in stark contrast with Fukui et al. (2014b) who claimed that $85 \%$ of lines of sight at essentially $|b|>15$ degrees have $\tau>0.5$ based on their comparison of $\tau_{353}$ and $N(H I)$.

\subsection{Spin Temperature}

Figure 5 (b) shows our estimated spin temperature which ranges from $\sim 5$ to $725 \mathrm{~K}$, with most CNM components having $T_{s}=10$ to $200 \mathrm{~K}$. The spin temperature distribution peaks at $\sim 50 \mathrm{~K}$, the median value is $49 \mathrm{~K}$. This is in excellent agreement with HT03 results based on 66 random lines of sight at $|b|>10^{\circ}$, as shown in the same figure. While we have a slightly smaller number of components relative to the HT03 study, the agreement between two studies is excellent over the full temperature range. In summary, the component spin temperature, for the predominantly CNM population we are tracing in absorption, is similar between a large angular area and a more focused

3C092

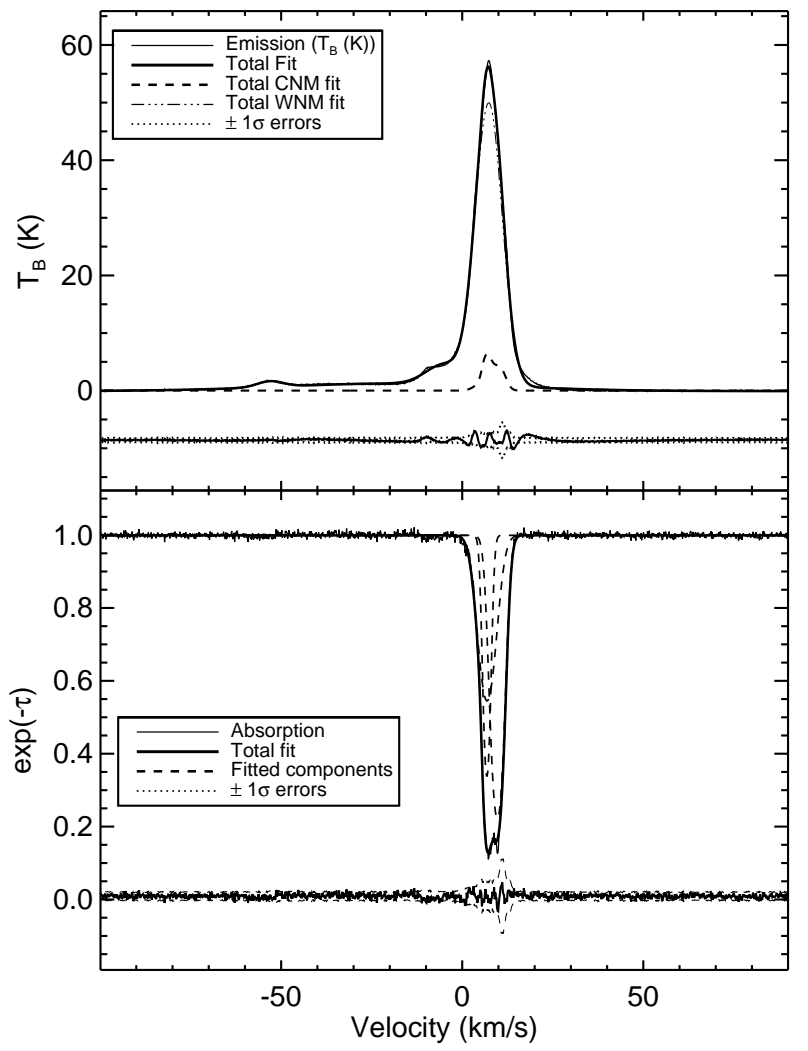

NV0157+28

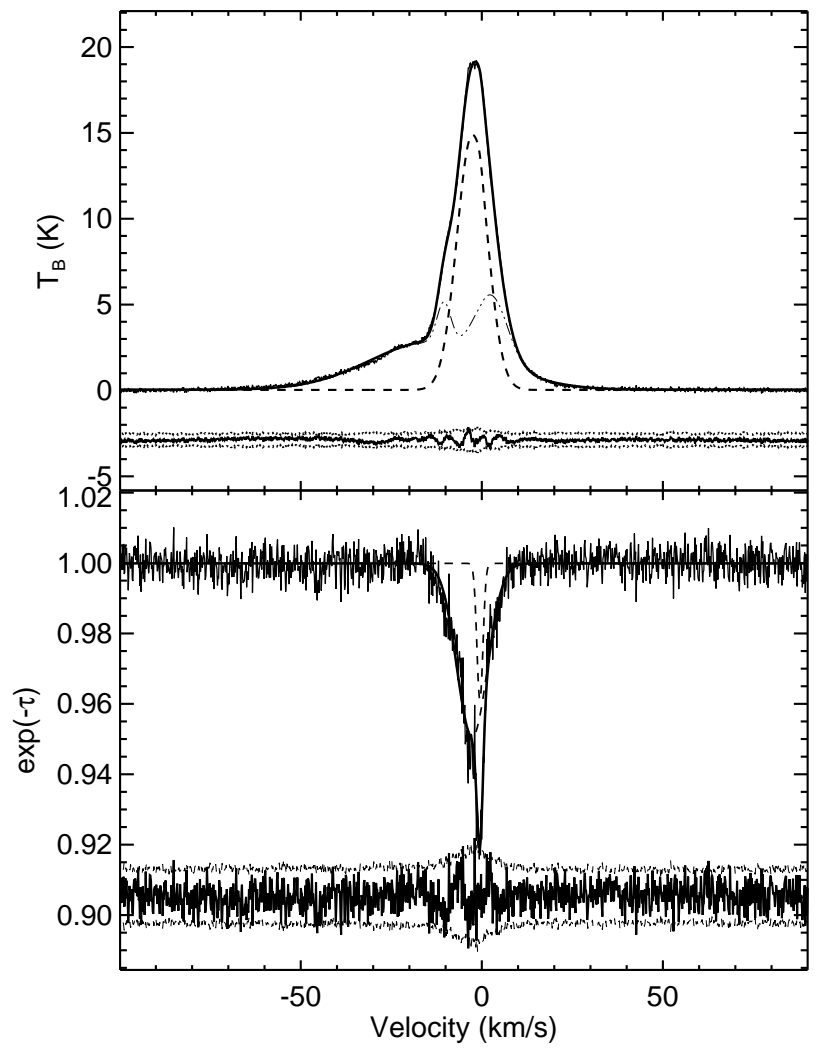

FIG. 4.- Example Gaussian fits to emission and absorption spectra. (Left) 3 C092 which is located behind the main body of Perseus, (Right) NV0157+28. See Figure 3 for a detailed description of the panels. 

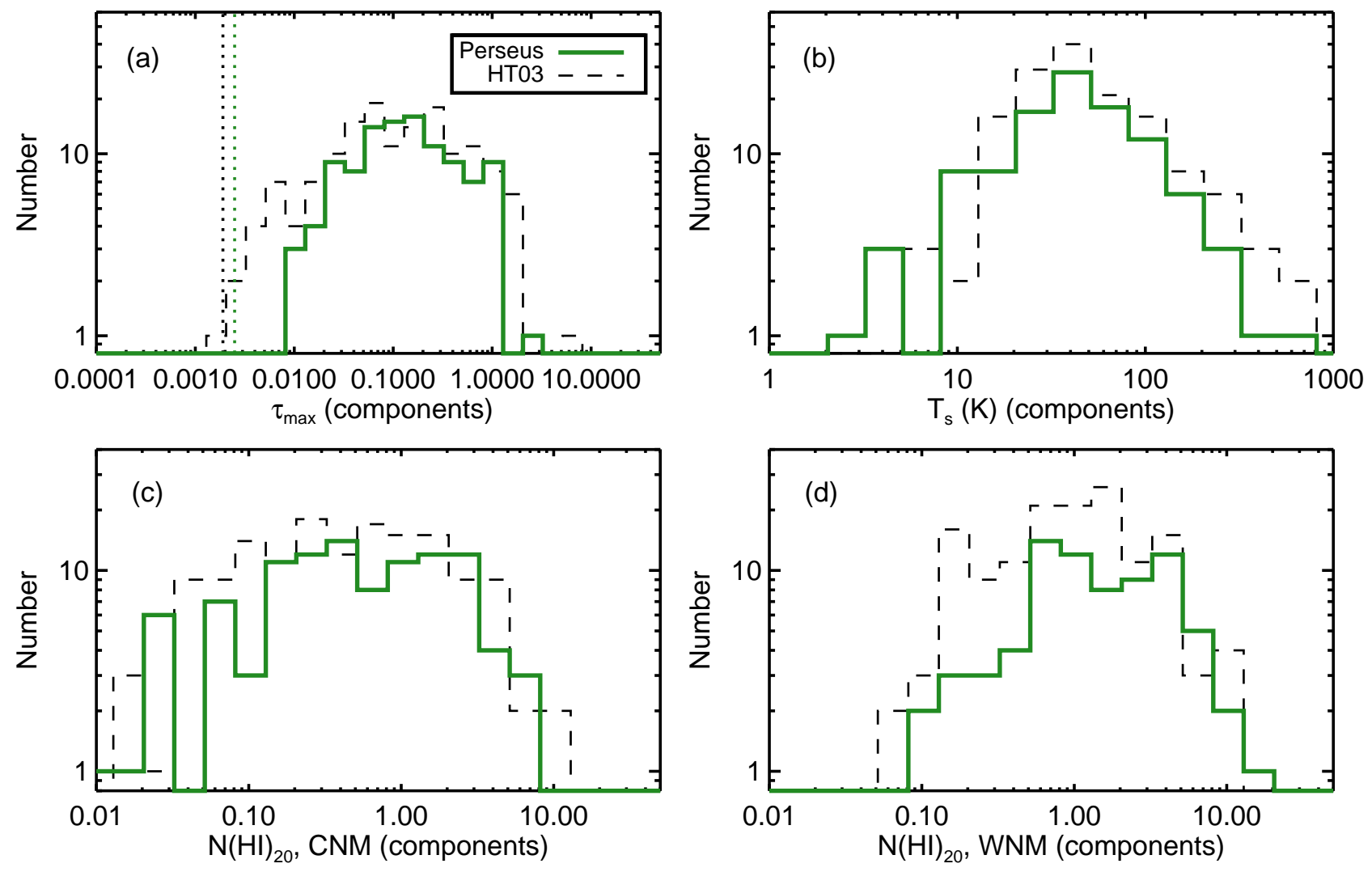

FIG. 5.- Histograms of Gaussian fit parameters for individual Gaussian components: (a) peak optical depth $\tau_{\max }$, (b) spin temperature, (c) the CNM column density (in units of $10^{20} \mathrm{~cm}^{-2}$ ), and (d) the WNM column density (in units of $10^{20} \mathrm{~cm}^{-2}$ ). Gaussian components from the HT03 survey at $|b|>10$ degrees are shown with a dashed black line for comparison. Dotted lines show the median sensitivity in optical depth for two studies. We assume here as the CNM all HI detected in absorption. 

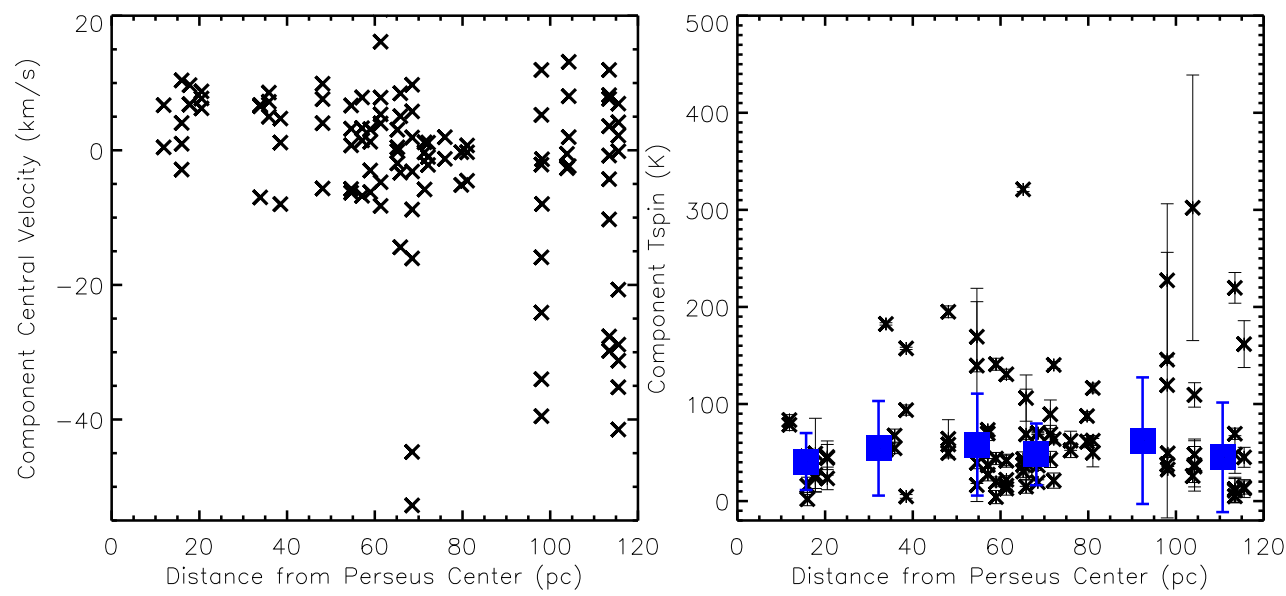

FIG. 6.- (Left) The central velocity of all Gaussian components showing that most components are found within -20 to $20 \mathrm{~km} \mathrm{~s} \mathrm{~s}^{-1}$ velocity range. (Right) The spin temperature of Gaussian components with velocity centroid within -20 and $20 \mathrm{~km} \mathrm{~s}$ shown as a function of distance from the Perseus center. Squares show median values of $T_{s}$ calculated over $20 \mathrm{pc}$ wide distance bins. The angular separation has been converted into linear distance assuming a distance of $300 \mathrm{pc}$.

area around Perseus.

In addition to HT03, one of our sources, 3C093.1, was observed by Andersson et al. (1992) who found only one absorption component and estimated its spin temperature of $41 \mathrm{~K}$. The line of sight to this source pierces through the main body of Perseus. We have fitted the HI absorption spectrum with three components, and their spin temperature is $45 \pm 13,44 \pm 17$ and $23 \pm 11$, respectively. The range of spin temperature in the direction of additional seven sources observed by Andersson, Roger, Wannier (1992) is $40-100 \mathrm{~K}$. This all shows that our derived temperatures are in general agreement with previous studies. Our mean $T_{s}$ is also in agreement with an estimate from Lee et al. (2012) of 60-75 K, where the equilibrium KMT09 model for the $\mathrm{H}_{2}$ fraction was fitted to observations, under the overarching assumption of the CNM and WNM co-existing in pressure equilibrium.

In stark contrast to Fukui et al. (2014b), we find the spin temperature distribution essentially identical to an average CNM temperature distribution for the Milky Way, e.g. HT03 or Strasser et al. (2007). Out of 107 absorptiondetected Gaussian components, $\sim 50 \%$ have $T_{s}<50 \mathrm{~K}$. There are three sources whose projected distance from the rough center of Perseus is less than $20 \mathrm{pc}$, and their mean spin temperature is $45 \mathrm{~K}$. The low spin temperature (20$40 \mathrm{~K}$ for $85 \%$ of data points) in Fukui et al. could be an artifact of neglecting to account for the "CO-dark" $\mathrm{H}_{2}$ gas in the $\tau_{353}-\mathrm{N}(\mathrm{HI})$ correlation, and the use of line-of-sight averaged properties (single spin temperature and optical depth).

In Figure 6 (left) we show the central velocity of all Gaussian CNM components which shows that most components have a central velocity between -20 and 20 $\mathrm{km} \mathrm{s}^{-1}$. In Figure 6 (right) we plot $T_{s}$ for all components within this velocity range, excluding components that are likely (based on their central velocity) not associated with Perseus. Squares show median $T_{s}$ over $20 \mathrm{pc}$ wide bins.
We do not find obvious variations of $T_{s}$ with the distance from the center of Perseus.

Spatial changes of $T_{s}$ across interstellar clouds have been claimed in the literature. Liljestrom \& Mattila (1988) mapped the HI distribution of a high latitude cloud and interpreted the observed increase in the line width as being due to an increase of $T_{s}$ by $\sim 30 \mathrm{~K}$. Andersson, Roger, Wannier (1992) performed radiative transfer modeling of HI observations of the B5 region in Perseus, considering internal stars and the effect of stellar winds on the spin temperature distribution. Their model suggests spin temperature of $40-50 \mathrm{~K}$ within the first $2 \mathrm{pc}$ from the central star cluster, and then an increase to $200-300 \mathrm{~K}$ out to $6-8$ pc from the core center. We do not find any evidence for a systematic change of $T_{s}$ radially from the Perseus center as shown in Figure 6. however we have large gaps in the background source coverage. A much tighter grid of $\mathrm{HI}$ absorption spectra within $50 \mathrm{pc}$ from the center would be important for future studies.

\subsection{HI column density and the CNM fraction}

Histograms of the CNM and WNM column densities derived for individual Gaussian components are shown in Figure 5 (c) and (d) as solid green, while the results from the HT03 survey are shown as dashed black. There is excellent agreement between two studie: 9 . Our median CNM column density is $6.0 \times 10^{19} \mathrm{~cm}^{-2}$, in comparison to $5.2 \times 10^{19} \mathrm{~cm}^{-2}$ by HT03. Our median WNM column density is $1.5 \times 10^{20} \mathrm{~cm}^{-2}$, in good agreement with $1.3 \times 10^{20}$ $\mathrm{cm}^{-2}$ estimated by HT03. Please note that both studies treated as the CNM all $\mathrm{HI}$ detected in absorption and no temperature selections were made to distinguish the CNM from the thermally unstable WNM. It is interesting to note that Figure 5 shows that the WNM has a more uniform column density, while the CNM column density varies more dramatically, from $10^{18}$ to $10^{21} \mathrm{~cm}^{-2}$.

In Figure 7 we show integrated CNM and WNM prop-

\footnotetext{
${ }^{9}$ To quantify this we have calculated cumulative distribution functions for $\tau_{\max }, T_{s}, N(H I)_{C N M}$ and $N(H I)_{W N M}$ to compare our results with HT03. The K-S test suggests that there is $83 \%$ and $72 \%$ probability that Perseus and HT03 $T_{s}$ and $N(H I)_{C N M}$ distributions were drawn from the same sample.
} 

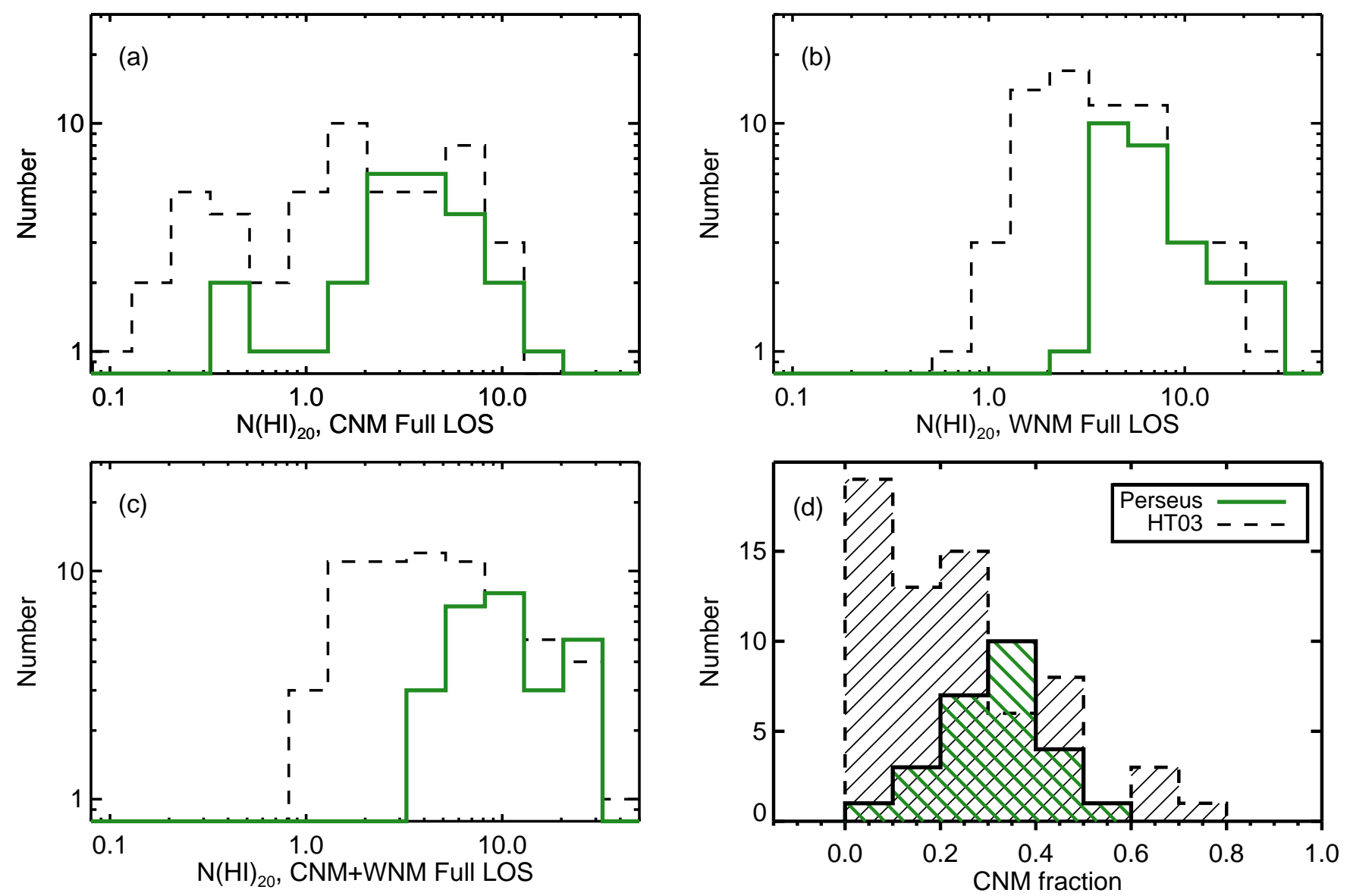

FIG. 7.- Histograms of basic properties calculated for each line of sight, from this study as solid green and from HT03 (for their $|b|>10^{\circ}$ sources) as dashed black: (from top left to bottom right) the CNM column density, the WNM column density, the CNM+WNM column density, and the CNM fraction (CNM/(CNM+WNM) column density). A $T_{s}$ threshold of $200 \mathrm{~K}$ was applied when selecting CNM components. 
erties for different lines of sight probed by our target background sources, as well as results from HT03 for their 66 random directions at $|b|>10^{\circ}$. As several of our CNM components have higher temperature likely more appropriate for the thermally unstable WNM (e.g. Kim et al. 2014), we have applied a temperature threshold of $T_{s}<200 \mathrm{~K}$ when calculating the CNM column density and the CNM fraction along the line of sight. The same threshold was applied for the HT03 data.

The main conclusion from this figure is that the line of sight properties in our study trace the upper range of the HT03 histograms. In terms of details, we find median CNM and WNM HI column density of $4.5 \times 10^{20} \mathrm{~cm}^{-2}$ and $7.8 \times 10^{20} \mathrm{~cm}^{-2}$, respectively. Both values are more than five times higher than the corresponding median values in HT03. The same applies to the total HI column density. To quantify the disagreement between our study and HT03 we have calculated cumulative distribution functions for all distributions in Figure 7. The K-S test suggests that there is $\lesssim 3 \%$ probability that Perseus and HT03 line-of-sight distributions were drawn from the same sample.

HT03 found a large number of sources with $\Sigma N(H I)_{C N M}=0$ as can be seen in Figure 7(d) where $\sim 15$ of HT03's sources did not have detectable CNM. Stanimirović \& Heiles (2005) and Stanimirović et al. (2007) showed that with $>4$ times longer integrations weak CNM features were detected in some of these directions. For each of our 26 sources we detect significant HI absorption lines with the CNM fraction being $>20 \%$ for 20 sources, the lowest CNM fraction we find is $1 \%$ and there is only one source with such low fraction. As the sensitivity of two studies is on average similar, our higher fraction of absorbing HI likely stems from the intrinsic properties of the Perseus region. Our median CNM fraction is 0.33 , in comparison to 0.22 in HT03 (after the same $200 \mathrm{~K}$ cut-off was applied to both studies). The above results strongly suggest that the Perseus region has a higher fraction of absorbing $\mathrm{HI}$ and a higher total HI column density relative to an average ISM field. The absorbing HI appears to contribute significantly to the total column density along almost every line of sight.

In summary, while properties of individual components are in excellent agreement with those of HT03, it appears that the Perseus region has a larger number of absorbing HI components relative to an average, random ISM field. This could explain the enhanced total HI column density and the fraction of the absorbing HI. Our results in particular for the CNM (and to a smaller degree for the WNM) and the total $N(H I)$ essentially trace the upper range of the corresponding distributions from HT03.

The CNM fraction, and especially its variations with interstellar environments, are poorly constrained observationally. In a comprehensive study of $290 \mathrm{HI}$ emission/absorption pairs, Dickey et al. (2009) showed that the radial dependence of the harmonic mean spin temperature, which is a product of the spin temperature and the CNM fraction, is flat across the Milky Way disk. Considering that Strasser et al. (2007) showed that spin temperature of the CNM is similar between the inner and outer Galaxy, this result implies a nearly constant CNM fraction out to $25 \mathrm{kpc}$. Our study of Perseus is the first hint that the CNM fraction in/around GMCs is likely higher than what is found in an average ISM field.

\subsection{What determines the CNM fraction?}

While the Perseus region has on average a higher CNM fraction relative to an average ISM field, as shown in Figure [7. interestingly almost all directions (25/26) in our study have the CNM fraction smaller than $50 \%$. We emphasize that this result is not an artifact of our applied $T_{s}<200 \mathrm{~K}$ cutoff. If we do not apply any temperature cutoff, the median CNM fraction is $35 \%$, and $23 / 26$ directions have a CNM fraction $<50 \%$.

In Figure 8 we show the CNM fraction as a function of the total HI column density for our sources as well as HT03 data. It is obvious that the CNM fraction never gets higher than $\sim 80 \%$ (for both studies). This shows that there are no lines of sight without the WNM, even in the directions where the CNM hugely dominates the WNM fraction is at least $\sim 20 \%$. Although the scatter in this figure is large, the CNM fraction appears to increase from 0 to $\sim 40 \%$ at $N(H I) \sim 10^{21} \mathrm{~cm}^{-2}$, and then levels off (purple points show median values for Perseus observations). As pointed out by Heiles \& Troland (2003b), this transition occurs right around the column density required for shielding $\mathrm{H}_{2}$, suggesting that the CNM transitions into $\mathrm{H}_{2}$ as soon as the adequate shielding is achieved. This column density also agrees with Lee et al. (2012) who showed that the HI-to- $\mathrm{H}_{2}$ transition (defined as having a $\mathrm{H}_{2}$ fraction of 0.25$)$ occurs in Perseus at $N(H I)=(6-12) \times 10^{20}$ $\mathrm{cm}^{-2}$.

In Figure 9 (top) crosses show the CNM fraction as a function of the column density weighted average spin temperature along the line of sight. A recent study by Kim et al. (2014), which produced synthetic HI spectra based on their 3D hydrodynamic simulations of a Milky Way-like disk, suggested that for the observed $T_{s}<400 \mathrm{~K}$ the CNM fraction is proportional to the inverse of $T_{s}$. While their synthetic spectra represent random directions, most of the simulated data are located between $50 \mathrm{~K} / T_{s}$ and $100 \mathrm{~K} / \mathrm{T}_{s}$ lines. Furthermore, for the observed $T_{s}<200 \mathrm{~K}$ the simulated CNM fraction ranges between $40 \%$ and $70 \%$, with a median value being $52 \%$ (97\% of simulated data points have a CNM fraction $<70 \%$, Kim et al. private communication). We overplot the $1 / T_{s}$ relation in the figure for three representative temperature values of 20,50 and 100 $\mathrm{K}$ (the simulation applied a CNM temperature cut-off of $T_{k}<184 \mathrm{~K}$, where $T_{k}$ is the true kinetic temperature ). To bracket most of our data points we need to expand the $T_{s}$ range to lower temperatures of $\lesssim 20 \mathrm{~K}$. With our observed CNM fraction being largely in the range of $10-50 \%$, we overlap with the $40-70 \%$ range expected by the simulation although, the simulated fraction is generally slightly higher than what we observe. Square symbols in this plot show the difference introduced in the CNM fraction when a $350 \mathrm{~K}$ threshold is applied (instead of $200 \mathrm{~K}$ ) to select the CNM. The difference is very small, only three data points are noticeably affected.

In the same figure (bottom panel) instead of using our calculated $T_{s}$ we follow exactly Kim et al. (2014) and calculate the observed temperature as the optical-depth weighted average spin temperature along the line of sight (equation (15) from Kim et al.). Most of our data points follow the $50 / T_{s}$ line, which agrees well with our median 
$T_{s}$ estimate, and is in excellent agreement with the Kim et al (2014) prediction. Considering that in the simulation the CNM fraction is known, while the observed CNM fraction is based on the Gaussian-decomposition $T_{s}$ derivation method, this excellent agreement is an indirect evidence that the observational method provides consistent and reliable CNM fractions.

While the optical-depth weighted average spin temperature (shown in the bottom panel) is on average higher than our column density weighted spin temperature (top panel), at the lowest temperatures the observed CNM fraction is in the $10-50 \%$ range, while the simulation suggests a CNM fraction of $40-70 \%$. The simulated fraction is slightly higher that what is observed, however, it is very encouraging to see that the simulated CNM fractions are so close to observations and that the observed CNM fraction follow the $50 / T_{s}$ prediction so closely. Considering that Kim et al. (2014) do not include interstellar chemistry, they likely slightly over-estimate the amount of cold $\mathrm{HI}$ as the conversion from atomic to molecular phase is not taking place in the simulation.

In summary, the CNM fraction in and close to Perseus is surprisingly low, being largely below $50 \%$ (median value of $30 \%$ ), even at the lowest observed temperature where HI absorption should be tracing only the CNM with essentially no confusion by the thermally unstable WNM. This is a somewhat surprising result as suggests that even close to the dense molecular clouds the CNM fraction (CNM/CNM+WNM column density) is never very high. As a consequence, this suggests that even lines of sight that probe deep inside the GMCs have of order of $50 \%$ contribution from the WNM (thermally unstable and/or stable). The geometry and the level of mixing of the CNM and WNM are still not understood; for example it is not clear if the WNM is located primarily in outer regions of the HI envelope, or is it being brought closer to the inner regions via turbulence. From the observational point of view, the HI absorption may not be tracing the densest $\mathrm{HI}$ regions as optical depth profiles may become saturated, like in the case of $4 \mathrm{C}+32.14$ which is the source we had to exclude from analysis due to its highly saturated HI absorption profile (this source is located behind the main body of Perseus). It will be important to investigate the CNM fraction using alternative tracers in the future, like CII and CI.

As the mixture of CNM and WNM phases exists in the diffuse ISM, Hennebelle \& Inutsuka (2006) asked the question of whether the WNM can persist deep inside molecular clouds. Considering that HI halos surround molecular clouds, interstellar turbulence will naturally mix in some HI with molecular gas. However, in about one cooling time it is expected that any WNM mixed with molecular gas will cool down if the internal pressure is about 10 times higher than the typical ISM pressure. Hennebelle \& Inutsuka (2006) showed that the dissipation of magnetic waves can provide substantial heating and therefore serve as an additional source of energy that can maintain the WNM inside even high-pressure molecular clouds.

\section{COMPARISON OF CO AND HI ABSORPTION SPECTRA}

To compare $\mathrm{HI}$ absorption with $\mathrm{CO}$ we use data from two surveys: the CO (1-0) emission data from the CfA survey at 8.4' resolution (Dame at al. 2001), and the integrated $\mathrm{CO}$ intensity $\left(W_{C O}\right)$ from Planck (Planck Collaboration et al. 2013) smoothed and regridded to match the CfA's angular resolution and pixel size. We extract CO spectra from Dame et al. and show $W_{C O}$ in Figure 10 (left) as black data points. The Dame et al. observed area covers 14 out of 26 sources. The dashed black line in this figure shows the median 1- $\sigma$ uncertainty on $W_{C O}$ calculated from the line-free channels. The results from Planck are shown in Figure10 (left and right) in blue, as well as their median 1- $\sigma$ uncertainty. We noticed that Dame et al.'s integrated intensity is systematically higher relative to the Planck data. A median scaling of 1.37 was applied on the Planck data to roughly match Dame et al. observations.

Figure 10 shows that 8 out of 26 sources have a clearly detected $\mathrm{CO}$ emission that is above 1- $\sigma$ uncertainty in both Dame at al. and Planck data. Almost all detections have the total HI column density $>10^{21} \mathrm{~cm}^{-2}$. Their CNM fraction ranges from $20 \%$ to $55 \%$. While $\mathrm{HI}$ absorption is detected in the case of all sources, 18 sources were not detected in $\mathrm{CO}$. Most non-detections pile up at $N(H I)<10^{21} \mathrm{~cm}^{-2}$ and likely probe diffuse HI regions. As shown in Figure 10 (right) where we use the Planck data for $E(B-V) \times 3.19$ as a measure of $A_{V}\left(R_{V}=3.19\right.$ was measured for Perseus star BD $+31^{\circ} 643$ by (Snow et al. 1994)), most non-detections have $A_{V}<1$. Lee et al. (2014) showed that in Perseus $A_{V} \sim 1 \mathrm{mag}$ is a necessary condition for the existence (shielding against photodissociation) of $\mathrm{CO}$. Considering all this, most $\mathrm{CO}$ non-detections probe diffuse $\left(A_{V}<1 \mathrm{mag}\right)$ regions without necessary shielding for $\mathrm{CO}$ formation.

However, there are $3 \mathrm{CO}$ non-detections with $N(H I)=10-35 \times 10^{20} \mathrm{~cm}^{-2}$, which probe regions with $A_{V} \sim 1 \mathrm{mag}$ and therefore likely contain $\mathrm{H}_{2}$, while $\mathrm{CO}$ could be just forming and still be underabundant. Considering that we detect $\mathrm{HI}$ absorption with large column density, yet no $\mathrm{CO}$ emission, these three positions are excellent candidates for probing the $\mathrm{CO}$-dark gas which contains $\mathrm{H}_{2}$ but not $\mathrm{CO}$. Interestingly, $\mathrm{CO}$ is detected both at lower and higher total $\mathrm{HI}$ column density relative to these non-detection, at $\lesssim 10^{21}$ and $>3 \times 10^{21} \mathrm{~cm}^{-2}$. The three sources are: 3C132, 3C093.1, and B20411+34. As shown in Figure 2, their HI absorption spectra have only components around $0 \mathrm{~km} / \mathrm{s}$ suggesting that a contamination from non-Perseus HI clouds can not be the reason for HI absorption detections without $\mathrm{CO}$ emission.

We now compare closely the kinematics of CO (from Dame et al.) and HI absorption of 8 sources with detected $\mathrm{CO}$ which have $A_{V} \gtrsim 1 \mathrm{mag}$ (Figure 11). One of the eight sources, 3C092, is particularly interesting as it is located right behind the main body of Perseus, this source has the highest integrated $\mathrm{CO}$ intensity $\left(>30 \mathrm{~K} \mathrm{~km} \mathrm{~s}^{-1}\right)$ and the CNM fraction of $\sim 0.4$. As shown in the figure, in most cases $\mathrm{CO}$ and $\mathrm{HI}$ absorption agree well in terms of velocity range and profile shapes, although there is a large diversity among sources. This suggests that HI in absorption appears to trace not just cloud envelopes but also central regions. In three cases (3C092, 3C108 and $4 \mathrm{C}+25.14) \mathrm{CO}$ emission and $\mathrm{HI}$ absorption cover the same velocity range. In the case of $3 \mathrm{C} 131,3 \mathrm{C} 133$ and $4 \mathrm{C}+27.14$, while the strongest $\mathrm{HI}$ absorption agrees well with the $\mathrm{CO}$ 


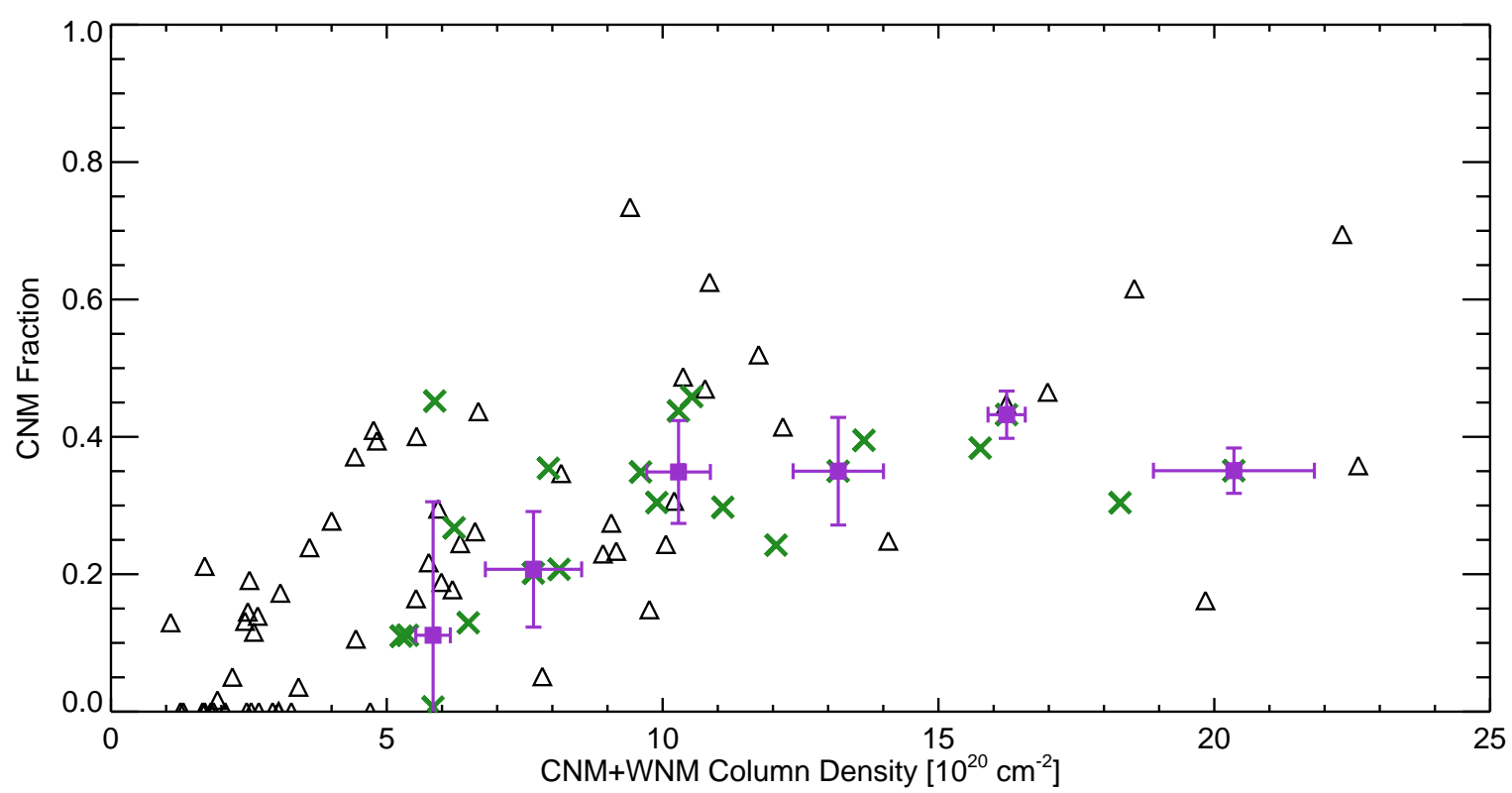

FIG. 8.- The CNM fraction as a function of the total HI column density, green crosses show our data and black triangles are from HT03. Purple points show median values with $1-\sigma$ scatter calculated for our observations only. To isolate CNM-only and exclude potentially thermally-unstable WNM we have applied a cutoff $T_{s}<200 \mathrm{~K}$ for both data sets.

emission peak, a weaker secondary component is seen at a velocity of $0 \mathrm{~km} / \mathrm{s}$ which is not detected in $\mathrm{CO}$, possibly due to low sensitivity. Only for two sources, $4 \mathrm{C}+30.04$ and $4 \mathrm{C}+33.10$ there is significant difference in that the $\mathrm{HI}$ absorption profile is broader than $\mathrm{CO}$ emission and a $\mathrm{CO}$ peak is found in the middle of the HI absorption profile.

We show in Figure 11 the corresponding spin temperature and the CNM column density of the HI component that is the closest in velocity to the CO peak. The spin temperature ranges from 30 to $80 \mathrm{~K}$, and the CNM column density of the component closest to $\mathrm{CO}$ ranges from 0.8 to $8 \times 10^{20} \mathrm{~cm}^{-2}$, which corresponds to the higher portion of the CNM column density measured for the whole population of CNM components in this study. On the other hand, the remaining CNM column density along these lines of sight ranges from $\sim 3 \times 10^{20}$ to $13 \times 10^{20} \mathrm{~cm}^{-2}$. All sources except $4 \mathrm{C}+25+14$ have the total HI column density $>10^{21} \mathrm{~cm}^{-2}\left(A_{V} \gtrsim 1 \mathrm{mag}\right)$, suggesting conditions suitable for formation of $\mathrm{CO}$ (and $\mathrm{H}_{2}$ ).

This generally good spectral agreement we find between $\mathrm{HI}$ absorption and $\mathrm{CO}$ emission contrasts results from studies of the diffuse molecular gas $\left(A_{V}<1 \mathrm{mag}\right)$, e.g. Liszt \& Lucas (1996); Liszt \& Pety (2012), where commonly $\mathrm{HI}$ absorption is more extended in velocity relative to $\mathrm{CO}$ emission, and especially it was noticed that $\mathrm{CO}$ emission tends to avoid the deepest HI absorption (in other words, $\mathrm{CO}$ was associated only with weaker $\mathrm{HI}$ absorption features). This is usually explained as the deepest HI absorption arising mainly from the CO-free cloud envelopes, while $\mathrm{CO}$ tracing the central regions. The eight directions we investigate here all trace regions with $A_{V} \gtrsim 1$ and are therefore likely probing equilibrium chemistry relative to $A_{V}<1$ likely largely non-equilibrium dominated regions.

It is generally expected that the CO-dark gas is found in uniform envelopes surrounding CO-bright molecular clouds (Wolfire et al. 2010). Numerical simulations by Smith et al. (2014) support this idea but show that COdark $\mathrm{H}_{2}$ may be asymmetric and not necessarily trace the outlines of CO-bright clouds. Fukui et al. (2014a) proposed that the CO-dark gas could be dominated by the optically thick HI. In addition, considering that envelopes are likely to have small velocity offsets relative to the CObright cloud regions, we would expect to see kinematically more extended $\mathrm{HI}$ absorption profiles around $\mathrm{CO}$ peaks. However, in eight directions where we have both $\mathrm{HI}$ absorption and $\mathrm{CO}$ emission spectra we generally find good agreement between the two. This suggests that in these directions $\mathrm{HI}$ absorption traces largely the central cloud regions where $\mathrm{CO}$ is bright, and to a smaller degree only the CO-dark cloud envelope. Of 26 directions there are only 3 cases with strong HI absorption and the total $N(H I)>10^{21} \mathrm{~cm}^{-2}$, but without CO emission.

Another interesting result from our study is that cold HI with high HI column density is clearly present deep inside CO-bright GMCs, suggesting that its importance for GMC evolution, and star formation, may be more significant than previously thought. The origin of cold HI deep inside GMCs, and its morphology (e.g. filamentary flows vs clumps vs diffuse distribution throughout GMCs) are not well understood. The cold HI could be brought deep into the clouds via circulation of neutral gas from outer regions due to turbulence (Hennebelle \& Inutsuka 2006), or could be a photodissociation product of $\mathrm{H}_{2}$. Tighter grids of $\mathrm{HI}$ absorption sources across and around GMCs are greatly needed to map out the distribution of cold HI and distinguish between various formation mechanisms.

\section{SUMMARY AND FUTURE WORK}



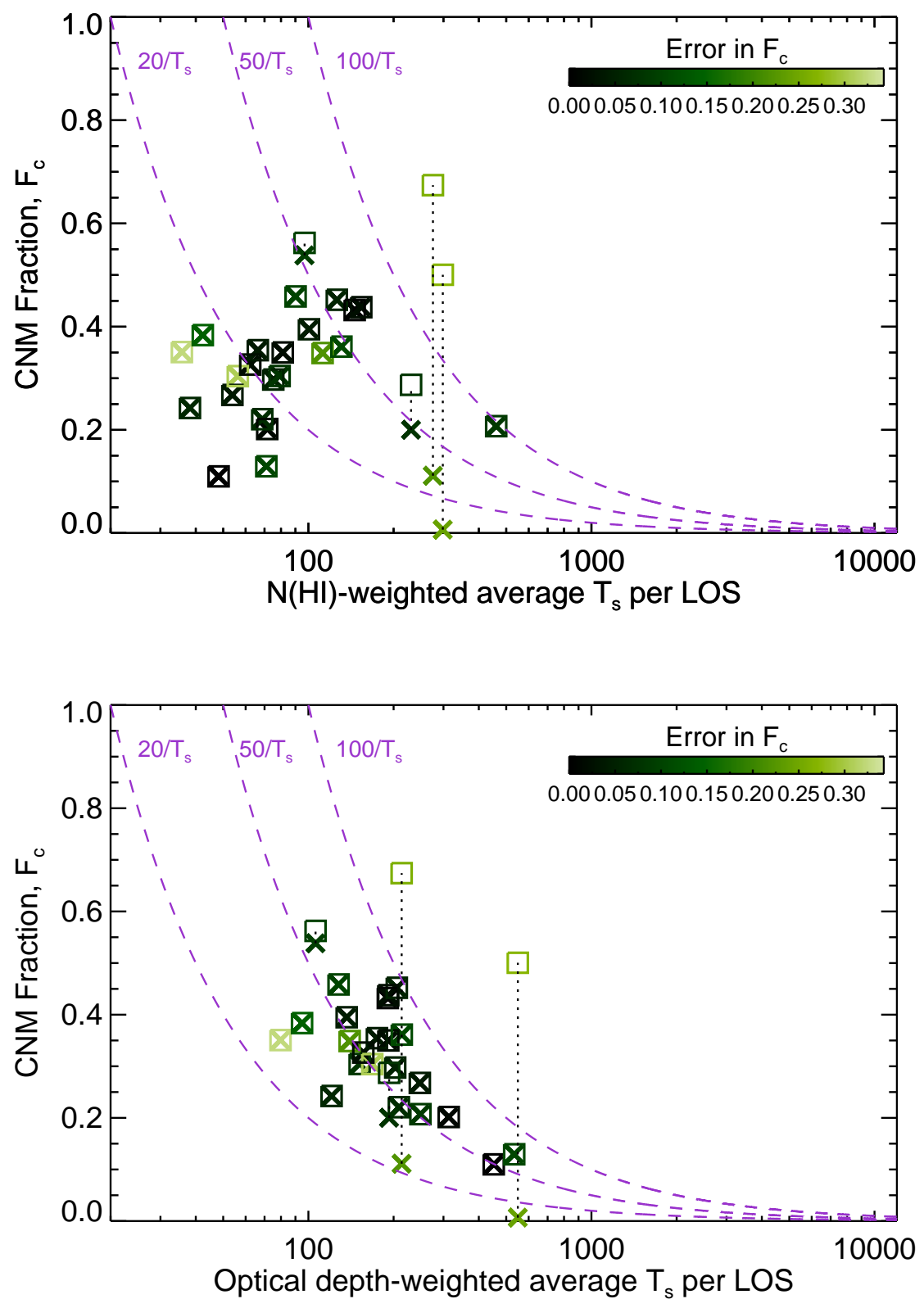

FIG. 9.- (Top) The CNM fraction as a function of the column density weighted $T_{s}$ along each line of sight for our observations (crosses). Propagated uncertainties, shown as a color bar, are just from the fitting of Gaussian components and do not include any systematic uncertainties inherent to the temperature derivation method. Crosses show the CNM fraction calculated when components with $T_{s}<200 \mathrm{~K}$ are considered as the CNM, while squares show the $T_{s}<350 \mathrm{~K}$ cut. The difference is very small and essentially only three sources have significantly changed their fraction. (Bottom) The CNM fraction as a function of the optical-depth weighted average $T_{s}$ (calculated using equation 15 in Kim et al. 2014), calculated using the optical depth and $T_{\text {exp }}$ profiles without Gaussian fitting. Again, squares show that the temperature cut does not affect a majority of our sources. 

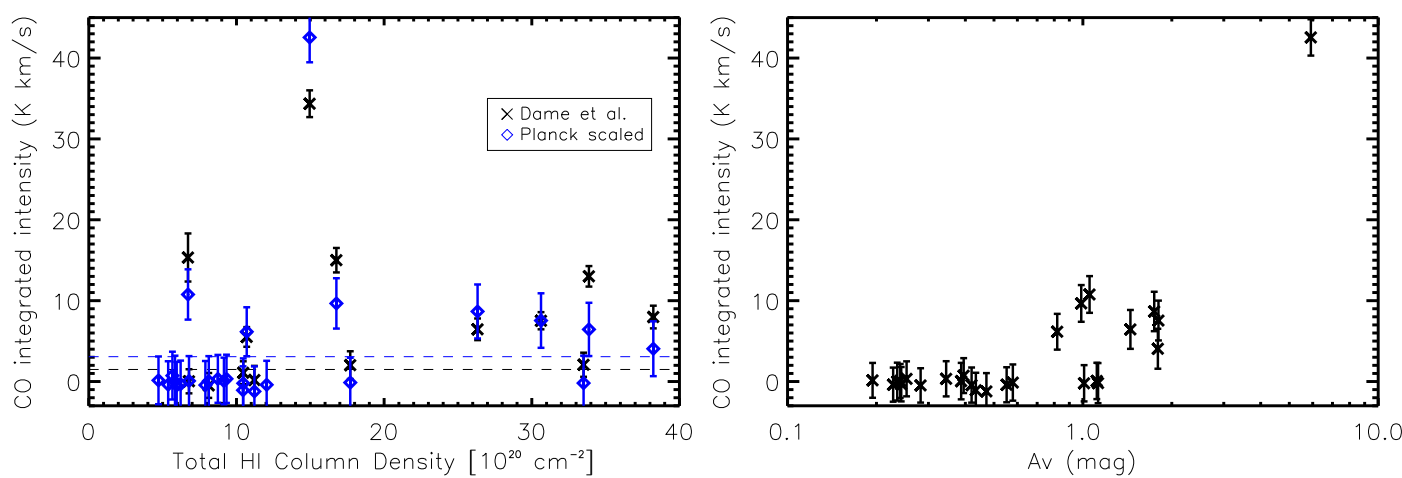

FIG. 10.- (Left) CO integrated intensity as a function of the total HI column density (CNM+WNM) in the direction of our 26 radio continuum sources. CO data from Dame et al. (2001) are shown in black for directions covered in this survey; the black dashed line shows the median 1- $\sigma$ uncertainty. CO data from Planck (Planck Collaboration et al. 2013) are shown in blue for all 26 sources after smoothing and regridding the $\mathrm{CO}$ integrated image to $8.4^{\prime}$ resolution to match Dame et al.; the dashed blue lines shows the median 1- $\sigma$ uncertainty. To match Planck and Dame et al. values a constant scaling factor of 1.37 had to be applied to Planck data. (Right) CO integrated intensity plotted as a function of $A_{V}$ from Planck.
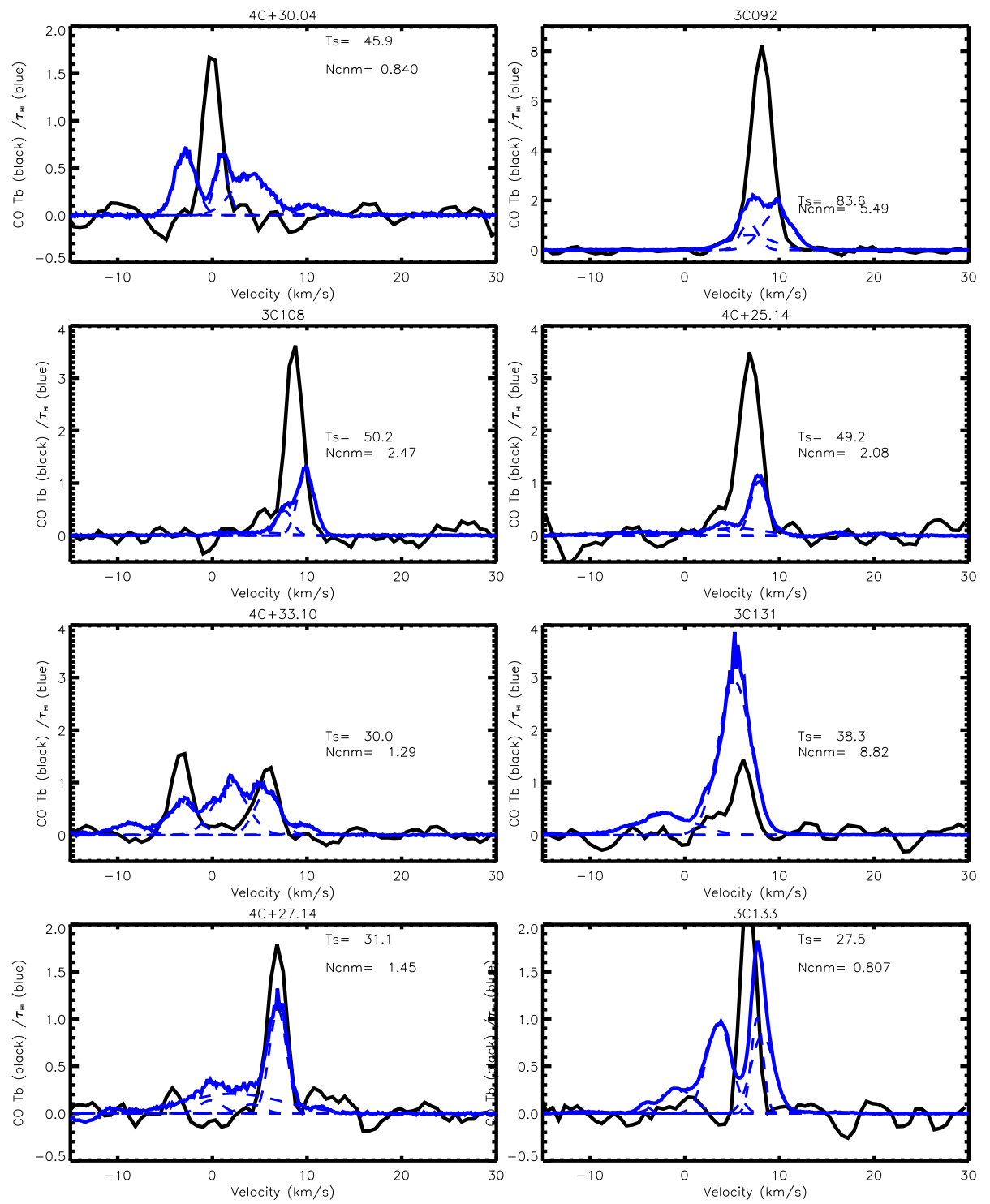

FIG. 11. - Comparison of CO emission (from Dame et al.) shown in black and HI absorption shown in blue. Each HI absorption profile has several Gaussian components shown as dashed lines. For each panel, properties of the HI absorption component that is closest in velocity to $\mathrm{CO}$ emission are listed in the top right corner: the corresponding spin temperature in $\mathrm{K}$, the corresponding CNM HI column density in $10^{20}$ $\mathrm{cm}^{-2}$, and the CNM fraction calculated for the whole line of sight. 
To investigate properties of cold HI in and around Perseus molecular cloud, and especially to investigate the role of cold $\mathrm{HI}$ in the shielding of $\mathrm{H}_{2}$ (Paper II), we have obtained and detected $\mathrm{HI}$ absorption in the direction of 26 background radio continuum sources. Using the corresponding $\mathrm{HI}$ emission spectra, and by employing a Gaussian decomposition of $\mathrm{HI}$ emission/absorption pairs, we have performed radiative transfer calculations to estimate $T_{s}$ and $\tau(v)$ for 107 individual Gaussian components. This method represents the most direct way of measuring spin temperature and optical depth.

The peak optical depth of individual Gaussian components ranges from $\sim 0.01$ to a few, with the median value of 0.16 . The spin temperature ranges from 10 to $725 \mathrm{~K}$, and peaks at $50 \mathrm{~K}$. The median values of the CNM and WNM column densities for individual components are $6 \times 10^{19}$ $\mathrm{cm}^{-2}$ and $1.5 \times 10^{20} \mathrm{~cm}^{-2}$, respectively. All properties of individual components for Perseus are in excellent agreement with those of HT03, who observed 66 random lines of sight at $|b|>10^{\circ}$. This suggests that individual cold $\mathrm{HI}$ components have similar properties between a focused field around a GMC and an average ISM field.

However, when all CNM and WNM components are summed along each line of sight, we find a significant difference relative to an average ISM field. The Perseus region has a higher fraction of absorbing $\mathrm{HI}$ and a higher total HI column density relative to an average ISM field, suggesting environmental differences. This result is the first observational evidence that the CNM fraction in/around GMCs is likely higher than what is found in an average ISM field. Interestingly, the median CNM and WNM HI column density along the line of sight are roughly similar around Perseus, $4.6 \times 10^{20} \mathrm{~cm}^{-2}$ vs $5.8 \times 10^{20} \mathrm{~cm}^{-2}$, while in the case of HT03 the WNM column density was twice higher than the CNM column density.

Our results for both the optical depth and spin temperature are in stark contrast to Fukui et al. (2014) who used Planck data and assumed that all of dust grains cooler than $22 \mathrm{~K}$ are mixed with the optically thick HI, suggesting that the amount of cold HI could be significant and even enough to explain all (or most) of the CO-dark gas. For $85 \%$ of their sky coverage they estimated $T_{s}=20-40$ $\mathrm{K}$ and $\tau_{\max }>0.5$. Considering all our Gaussian components, we find such high $\tau_{\max }$ only occasionally, with only $20 \%$ of components having $\tau_{\max }>0.5$. Considering whole optical depth profiles, $54 \%$ of directions have $\tau_{\max }>0.5$. Also, only $\sim 15 \%$ of lines of sight have a column density weighted average spin temperature lower than $40 \mathrm{~K}$. We suspect that Fukui et al. results are caused by the non ability to distinguish different gas components along the line of sight, as well as by assigning all of the cooler dust to HI without allowing for contribution of the molecular gas (bright or dark).

The mean spin temperature appears uniform over the radius of 10 to $120 \mathrm{pc}$ from the rough center of Perseus. Obtaining a tighter grid of $\mathrm{HI}$ absorption sources, and especially sampling better the inner $10 \mathrm{pc}$, in the future will be important to probe a potential radial increase in $T_{s}$ away from the cloud center as suggested by Andersson et al. (1992).

While the CNM fraction is on average higher around Perseus relative to a random ISM field, surprisingly it rarely exceeds $50 \%$. Even directions with the lowest $T_{s}<200 \mathrm{~K}$ clearly show the CNM fraction of $<50 \%$. It is highly encouraging to see that recent numerical simulations by Kim et al. (2014) produce the CNM fractions reasonably close to observations, $40-70 \%$, and also predict that the CNM fraction is inversely proportional to the optical-depth weighted average $T_{s}$, which is in excellent agreement with observations. Further inclusion of interstellar chemistry, and the HI-to- $\mathrm{H}_{2}$ conversion, will likely fine-tune the simulated fractions and bring them even closer to observations. Our results suggest that even directions that probe deep inside molecular clouds do not have high CNM fractions (e.g. > 50\%). This could result from extended WNM envelopes of GMCs, and/or significant mixing of CNM and WNM throughout GMCs caused by interstellar turbulence or accretion flows. While the low CNM fraction in/around GMCs requires further theoretical work, at high column densities the HI lines are likely to become saturated and therefore poorly trace the densest and coldest regions of GMCs. It is therefore also important to observationally test usefulness of additional tracers of neutral gas inside GMCs, e.g. CI and CII.

Finally, we have compared $\mathrm{HI}$ absorption with $\mathrm{CO}$ emission for our 26 directions and found that $8 / 26$ have detected CO. Out of the remaining 18, 15 directions probe diffuse regions with $A_{V}<1 \mathrm{mag}$ and likely do not have enough shielding for $\mathrm{CO}$ formation. Only $3 / 26$ directions have $N(H I)>10^{21} \mathrm{~cm}^{-2}\left(A_{V} \gtrsim 1 \mathrm{mag}\right)$, and therefore probe conditions suitable for $\mathrm{CO}$ formation, yet have no detected $\mathrm{CO}$ emission. These directions therefore likely contain molecular gas but not $\mathrm{CO}$ and are representative of so called CO-dark gas. Eight directions with detected CO have $N(H I)>10^{21} \mathrm{~cm}^{-2}, A_{V}>1 \mathrm{mag}$, and good kinematic agreement between $\mathrm{HI}$ absorption and $\mathrm{CO}$ emission spectra. All of this suggests that these lines of sight probe largely central CO-bright regions, confirming the existence of cold HI deep inside GMCs. However, future observations of a tighter grid of background sources are necessary to map out the distribution of cold HI around GMCs and its origin.

We sincerely thank telescope operators at the Arecibo Observatory for their help in conducting these observations. The Arecibo Observatory is operated by SRI International under a cooperative agreement with the National Science Foundation (AST-1100968), and in alliance with Ana G. Méndez-Universidad Metropolitana, and the Universities Space Research Association. We are extremely grateful to Chang-Goo Kim and Eve Ostriker for extensive discussions and for providing detailed CNM fractions from Kim et al. (2014) for comparison with observations. We acknowledge stimulating discussions with Robert Lindner and Brian Babler, and thank Elijah Bernstein-Cooper for extracting Planck images around Perseus. We also thank an anonymous referee for emphasizing the importance of detailed chemistry in neutral gas estimates. We are grateful to John Dickey for stressing the importance of background diffuse emission in spin temperature calculations. S.S. thanks the Department of Astronomy at the Faculty of Mathematics, Belgrade University for their kind hospitality during the final stage of manuscript preparation. This work was supported by the NSF Early Career Devel- 
opment (CAREER) Award AST-1056780. M.-Y. Lee acknowledges support from the DIM ACAV of the Region Ile de France. We also acknowledge the NSF REU grant AST-
1004881 which funded summer research of Jesse Miller. The use of "Karma" visualization software (Gooch 1996) is gratefully acknowledged.

\section{REFERENCES}

Andersson, B.-G., Roger, R. S., \& Wannier, P. G. 1992, A\&A, 260, 355

Andersson, B.-G. \& Wannier, P. G. 1993, ApJ, 402, 585

Audit, E. \& Hennebelle, P. 2005, A\&A, 433, 1

Bigiel, F., Leroy, A., Walter, F., et al. 2008, AJ, 136, 2846

Blitz, L., Fukui, Y., Kawamura, A., et al. 2007, Protostars and Planets V, 81

Blitz, L. \& Rosolowsky, E. 2004, ApJ, 612, L29

Chieze, J. P. \& Pineau Des Forets, G. 1989, A\&A, 221, 89

Clark, P. C., Glover, S. C. O., Klessen, R. S., \& Bonnell, I. A. 2012, MNRAS, 424, 2599

Condon, J. J., Cotton, W. D., Greisen, E. W., et al. 1998, AJ, 115, 1693

Dame, T. M., Hartmann, D., \& Thaddeus, P. 2001, ApJ, 547, 792

Dickey, J. M., Strasser, S., Gaensler, B. M., et al. 2009, ApJ, 693, 1250

Elmegreen, B. G. 1993, ApJ, 411, 170

Fukui, Y. \& Kawamura, A. 2010, ARA\&A, 48, 547

Fukui, Y., Kawamura, A., Wong, T., et al. 2009, ApJ, 705, 144

Fukui, Y., Okamoto, R., Yamamoto, H., et al. 2014a, astroph/1401.7398, submitted to ApJ

Fukui, Y., Torii, K., Onishi, T., et al. 2014b, astro-ph/1403.0999, submitted to ApJ

Gibson, S. J., Taylor, A. R., Higgs, L. A., \& Dewdney, P. E. 2000, ApJ, 540, 851

Goldbaum, N. J., Krumholz, M. R., Matzner, C. D., \& McKee, C. F. 2011, ApJ, 738, 101

Goldsmith, P. F. \& Li, D. 2005, ApJ, 622, 938

González Hernández, J. I., Iglesias-Groth, S., Rebolo, R., et al. 2009, ApJ, 706, 866

Gooch, R. 1996, Astronomical Data Analysis Software and Systems $\mathrm{V}, 101,80$

Goodman, A. A. \& Heiles, C. 1994, ApJ, 424, 208

Haslam, C. G. T., Stoffel, H., Salter, C. J., \& Wilson, W. E. 1982 , A\&AS, 47,

Heiles, C. \& Troland, T. H. 2003a, ApJS, 145, 329

-. 2003b, ApJ, 586, 1067

Heitsch, F., Burkert, A., Hartmann, L. W., Slyz, A. D., \&

Devriendt, J. E. G. 2005, Ap.J, 633, L113

Hennebelle, P. \& Inutsuka, S.-i. 2006, ApJ, 647, 404

Herbig, G. H. \& Jones, B. F. 1983, AJ, 88, 1040

Kalberla, P. M. W., Dedes, L., Arnal, E. M., et al. 2005, in ASP Conf. Ser. 331: Extra-Planar Gas, 81

Kim, C.-G., Ostriker, E. C., \& Kim, W.-T. 2014, ApJ, 786, 64
Kim, W.-T. \& Ostriker, E. C. 2006, ApJ, 646, 213

Knapp, G. R. 1974, AJ, 79, 527

Krumholz, M. R., McKee, C. F., \& Tumlinson, J. 2009, ApJ, 693, 216

Lada, C. J., Lombardi, M., \& Alves, J. F. 2010, ApJ, 724, 687

Lee, M.-Y., Stanimirović, S., Douglas, K. A., et al. 2012, ApJ, 748,

Li, D. \& Goldsmith, P. F. 2003, ApJ, 585, 823

Liljestrom, T. \& Mattila, K. 1988, A\&A, 196, 243

Liszt, H. \& Lucas, R. 1996, A\&A, 314, 917

Liszt, H. S. \& Pety, J. 2012, A\&A, 541, A58

McKee, C. F. \& Ostriker, J. P. 1977, ApJ, 218, 148

Miville-Deschênes, M.-A. \& Lagache, G. 2005, ApJS, 157, 302

Peek, J. E. G., Heiles, C., Douglas, K. A., et al. 2011, ApJS, 194, 20

Pineda, J. E., Caselli, P., \& Goodman, A. A. 2008, ApJ, 679, 481

Planck Collaboration, Ade, P. A. R., Aghanim, N., et al. 2013, astro-ph/1303.5073, submitted to A\&A

Ridge, N. A., Di Francesco, J., Kirk, H., 2006, AJ, 131, 2921

Sancisi, R., Goss, W. M., Anderson, C., Johansson, L. E. B., \& Winnberg, A. 1974, A\&A, 35, 445

Schruba, A., Leroy, A. K., Walter, F., et al. 2011, AJ, 142, 37

Shetty, R., Kelly, B. C., Rahman, N., et al. 2014, MNRAS, 437, L61

Shu, F. H. 1973, American Scientist, 61, 524

Smith, R. J., Glover, S. C. O., Clark, P. C., Klessen, R. S., \& Springel, V. 2014, MNRAS, 441, 1628

Snow, T. P., Hanson, M. M., Seab, G. C., \& Saken, J. M. 1994, ApJ, 420, 632

Spitzer, Jr., L. \& Jenkins, E. B. 1975, ARA\&A, 13, 133

Stanimirović, S. \& Heiles, C. 2005, ApJ, 631, 371

Stanimirović, S., Heiles, C., \& Kanekar, N. 2007, in Astronomical Society of the Pacific Conference Series, Vol. 365, SINS Small Ionized and Neutral Structures in the Diffuse Interstellar Medium, ed. M. Haverkorn \& W. M. Goss, 22

Stanimirović, S., Putman, M., Heiles, C., et al. 2006, ApJ, 653, 1210

Strasser, S. T., Dickey, J. M., Taylor, A. R., et al. 2007, AJ, 134, 2252

Wannier, P. G., Lichten, S. M., Andersson, B.-G., \& Morris, M. 1991, ApJS, 75, 987

Wannier, P. G., Lichten, S. M., \& Morris, M. 1983, ApJ, 268, 727

Wolfire, M. G., Hollenbach, D., \& McKee, C. F. 2010, ApJ, 716, 1191

Wolfire, M. G., McKee, C. F., Hollenbach, D., \& Tielens, A. G. G. M. 2003, Ap.J, 587, 278

Wong, T. \& Blitz, L. 2002, ApJ, 569, 157

\begin{tabular}{|c|c|c|c|c|c|c|c|c|}
\hline Source & $\begin{array}{l}\mathrm{T}_{B} \\
(\mathrm{~K}) \\
(1)\end{array}$ & $\begin{array}{c}\mathrm{V}_{\mathrm{LSR}} \\
\left(\mathrm{km} \mathrm{s}^{-1}\right) \\
(2)\end{array}$ & $\begin{array}{c}\Delta \mathrm{V} \\
\left(\mathrm{km} \mathrm{s}^{-1}\right) \\
(3)\end{array}$ & (4) & $\begin{array}{c}\mathrm{T}_{s} \\
(\mathrm{~K}) \\
(5)\end{array}$ & $\begin{array}{c}\mathrm{T}_{\mathrm{k}, \max } \\
(\mathrm{K}) \\
(6)\end{array}$ & $\begin{array}{c}\mathrm{N}(\mathrm{HI})_{20} \\
\left(\mathrm{~cm}^{-2}\right) \\
(7)\end{array}$ & $\bar{F}$ or $O$ \\
\hline $3 \mathrm{C} 067$ & $1.88 \pm 0.06$ & $-25.8 \pm 0.1$ & $4.71 \pm 0.18$ & 0.0057 & 331. & 835 & 0.17 & 1.0 \\
\hline $3 \mathrm{C} 067$ & $1.95 \pm 0.00$ & $-11.6 \pm 0.4$ & $41.30 \pm 0.64$ & 0.0045 & 438. & 2077 & 1.27 & 0.0 \\
\hline $3 \mathrm{C} 067$ & 5.34 & $-5.8 \pm 0.1$ & $4.81 \pm 0.18$ & $0.10 \pm 0.002$ & $69.10 \pm 8.88$ & 325 & 0.62 & 2 \\
\hline $3 \mathrm{C} 067$ & $21.02 \pm 0.35$ & $-2.3 \pm 0.2$ & $9.75 \pm 0.17$ & 0.0169 & 1254. & 861 & 3.99 & 0.5 \\
\hline $3 \mathrm{C} 067$ & 23.05 & $-0.3 \pm 0.0$ & $2.14 \pm 0.04$ & $0.41 \pm 0.008$ & $42.88 \pm 8.28$ & 607 & 0.74 & 0 \\
\hline $3 \mathrm{C} 067$ & 17.71 & $1.2 \pm 0.1$ & $5.99 \pm 0.13$ & $0.19 \pm 0.005$ & $89.34 \pm 14.82$ & 489 & 1.94 & 1 \\
\hline $3 \mathrm{C} 067$ & $3.41 \pm 0.19$ & $8.3 \pm 0.2$ & $6.28 \pm 0.31$ & 0.0005 & 6748. & 485 & 0.41 & 0.0 \\
\hline $3 \mathrm{C} 068.2$ & $1.24 \pm 0.04$ & $-19.8 \pm 1.4$ & $30.94 \pm 2.61$ & 0.0089 & 141. & 20919 & 0.55 & 0.0 \\
\hline $3 \mathrm{C} 068.2$ & 5.09 & $-6.2 \pm 0.0$ & $2.58 \pm 0.07$ & $0.27 \pm 0.000$ & $19.75 \pm 8.88$ & 144 & 0.27 & 0 \\
\hline $3 \mathrm{C} 068.2$ & -0.09 & $-3.0 \pm 0.1$ & $2.63 \pm 0.24$ & $0.09 \pm 0.004$ & $4.11 \pm 8.28$ & 151 & 0.02 & 3 \\
\hline $3 \mathrm{C} 068.2$ & $20.81 \pm 1.16$ & $-2.7 \pm 0.1$ & $10.42 \pm 0.25$ & 0.0147 & 1422. & 2370 & 4.22 & 0.0 \\
\hline $3 \mathrm{C} 068.2$ & 31.03 & $1.3 \pm 0.0$ & $2.04 \pm 0.05$ & $0.90 \pm 0.040$ & $43.82 \pm 14.82$ & 91 & 1.57 & 2 \\
\hline $3 \mathrm{C} 068.2$ & 16.32 & $3.1 \pm 0.6$ & $3.88 \pm 0.62$ & $0.11 \pm 0.017$ & $140.94 \pm 5.04$ & 328 & 1.15 & 1 \\
\hline $3 \mathrm{C} 068.2$ & $3.44 \pm 0.67$ & $5.6 \pm 2.1$ & $15.15 \pm 1.97$ & 0.0194 & 179. & 5017 & 0.96 & 1.0 \\
\hline 3C092 & $1.01 \pm 0.06$ & $-53.1 \pm 0.3$ & $8.43 \pm 0.67$ & 0.0117 & 86. & 119698 & 0.12 & 0.0 \\
\hline 3С092 & $1.32 \pm 0.04$ & $-18.6 \pm 0.9$ & $74.01 \pm 2.51$ & 0.0059 & 224. & 3173 & 1.18 & 0.0 \\
\hline 3С092 & $3.45 \pm 0.06$ & $-4.9 \pm 0.2$ & $12.05 \pm 0.48$ & 0.0031 & 1118. & 1661 & 0.81 & 0.0 \\
\hline
\end{tabular}


S. Stanimirović et al.

\begin{tabular}{|c|c|c|c|c|c|c|c|c|}
\hline Source & $\begin{array}{l}\mathrm{T}_{B} \\
(\mathrm{~K}) \\
(1)\end{array}$ & $\begin{array}{c}\mathrm{V}_{\mathrm{LSR}} \\
\left(\mathrm{km} \mathrm{s}^{-1}\right) \\
(2)\end{array}$ & $\begin{array}{c}\Delta V \\
\left(\mathrm{~km} \mathrm{~s}^{-1}\right) \\
(3)\end{array}$ & $(4)$ & $\begin{array}{l}\mathrm{T}_{s} \\
(\mathrm{~K}) \\
(5)\end{array}$ & $\begin{array}{c}\mathrm{T}_{\mathrm{k}, \max } \\
(\mathrm{K}) \\
(6)\end{array}$ & $\begin{array}{c}\mathrm{N}(\mathrm{HI})_{20} \\
\left(\mathrm{~cm}^{-2}\right) \\
(7)\end{array}$ & $\overline{F \text { or } O}$ \\
\hline $3 \mathrm{C} 092$ & 6.36 & $6.8 \pm 0.0$ & $2.22 \pm 0.06$ & $1.08 \pm 0.040$ & $26.89 \pm 8.28$ & 151 & 1.26 & 2 \\
\hline 3C092 & 6.36 & $6.9 \pm 0.5$ & $5.56 \pm 0.35$ & $0.61 \pm 0.114$ & $49.09 \pm 8.88$ & 144 & 3.23 & 1 \\
\hline 3C092 & $49.81 \pm 0.62$ & $7.3 \pm 0.0$ & $8.72 \pm 0.03$ & 0.0025 & 19811. & 328 & 8.46 & 1.0 \\
\hline 3C092 & 4.39 & $9.7 \pm 0.1$ & $3.82 \pm 0.12$ & $1.50 \pm 0.158$ & $23.70 \pm 14.82$ & 91 & 2.65 & 0 \\
\hline $3 \mathrm{C} 093.1$ & $1.86 \pm 0.10$ & $-21.2 \pm 0.2$ & $9.89 \pm 0.69$ & 0.0048 & 386. & 1240 & 0.36 & 0.0 \\
\hline 3С093.1 & $4.37 \pm 0.07$ & $-13.1 \pm 0.3$ & $47.01 \pm 0.74$ & 0.0016 & 2702. & 328 & 3.60 & 0.0 \\
\hline 3С093.1 & 36.62 & $6.2 \pm 0.0$ & $1.62 \pm 0.09$ & $0.41 \pm 0.031$ & $44.35 \pm 8.28$ & 151 & 0.57 & 0 \\
\hline 3С093.1 & $33.75 \pm 0.59$ & $7.2 \pm 0.0$ & $7.53 \pm 0.06$ & 0.0031 & 11047. & 48303 & 4.95 & 0.0 \\
\hline 3С093.1 & 35.18 & $7.7 \pm 0.0$ & $4.45 \pm 0.04$ & $1.19 \pm 0.043$ & $45.36 \pm 8.88$ & 144 & 4.67 & 2 \\
\hline 3С093.1 & 35.58 & $8.8 \pm 0.0$ & $1.36 \pm 0.03$ & $1.30 \pm 0.036$ & $23.35 \pm 14.82$ & 91 & 0.80 & 1 \\
\hline $3 \mathrm{C} 108$ & $1.91 \pm 0.05$ & $-24.1 \pm 0.9$ & $29.33 \pm 1.70$ & 0.0045 & 425. & 18806 & 0.92 & 0.0 \\
\hline 3C108 & 1.55 & $-5.7 \pm 0.5$ & $4.73 \pm 1.19$ & $0.01 \pm 0.002$ & $64.12 \pm 8.88$ & 144 & 0.07 & 2 \\
\hline $3 \mathrm{C} 108$ & $21 \pm 0.18$ & $-0.7 \pm 0.1$ & $4.69 \pm 0.14$ & 0.0066 & 792. & 481 & 0.48 & 1.0 \\
\hline $3 \mathrm{C} 108$ & 12.88 & $4.0 \pm 0.3$ & $7.52 \pm 0.69$ & $0.06 \pm 0.002$ & $94.98 \pm 8.28$ & 151 & 1.82 & 1 \\
\hline $3 \mathrm{C} 108$ & $76 \pm 0.19$ & $6.7 \pm 0.3$ & $23.59 \pm 0.31$ & 0.0007 & 13061. & 12158 & 4.42 & 0.0 \\
\hline 3C108 & 29.59 & $7.6 \pm 0.0$ & $1.99 \pm 0.05$ & $0.47 \pm 0.009$ & $58.26 \pm 14.82$ & 91 & 1.07 & 0 \\
\hline $3 \mathrm{C} 108$ & 37.55 & $9.9 \pm 0.0$ & $2.10 \pm 0.02$ & $1.20 \pm 0.010$ & $49.52 \pm 5.04$ & 328 & 2.44 & 3 \\
\hline $3 \mathrm{C} 131$ & 16.27 & $-39.5 \pm 0.0$ & $3.74 \pm 0.09$ & $0.16 \pm 0.003$ & $100.90 \pm 8.88$ & 144 & 1.15 & 2 \\
\hline $3 \mathrm{C} 131$ & 3.41 & $-34.0 \pm 0.1$ & $2.00 \pm 0.33$ & $0.03 \pm 0.004$ & $75.15 \pm 8.28$ & 151 & 0.09 & 3 \\
\hline 3C131 & 2.28 & $-24.1 \pm 0.2$ & $3.37 \pm 0.50$ & $0.03 \pm 0.003$ & $114.31 \pm 14.82$ & 91 & 0.20 & 4 \\
\hline $3 \mathrm{C} 131$ & $9.18 \pm 0.15$ & $-18.5 \pm 0.3$ & $53.41 \pm 0.68$ & 0.0079 & 1163. & 745 & 8.82 & 0.0 \\
\hline 3C131 & 4.62 & $-15.9 \pm 0.3$ & $6.76 \pm 0.70$ & $0.03 \pm 0.002$ & $227.36 \pm 5.04$ & 328 & 0.89 & 1 \\
\hline $3 \mathrm{C} 131$ & $7.18 \pm 0.26$ & $-9.6 \pm 0.1$ & $4.94 \pm 0.21$ & 0.0036 & 2023. & 745 & 0.69 & 0.0 \\
\hline 3C131 & 46.95 & $-2.1 \pm 0.0$ & $7.31 \pm 0.10$ & $0.38 \pm 0.003$ & $145.62 \pm 6.75$ & 62335 & 7.99 & 0 \\
\hline $3 \mathrm{C} 131$ & 46.76 & $5.2 \pm 0.0$ & $4.04 \pm 0.02$ & $2.93 \pm 0.028$ & $37.71 \pm 6.26$ & 534 & 8.68 & 5 \\
\hline $3 \mathrm{C} 131$ & $45.98 \pm 1.89$ & $5.9 \pm 0.0$ & $5.84 \pm 0.10$ & 0.0234 & 1985. & 745 & 5.23 & 0.0 \\
\hline $3 \mathrm{C} 131$ & 6.36 & $11.9 \pm 0.2$ & $2.65 \pm 0.54$ & $0.02 \pm 0.004$ & $119.41 \pm 6.19$ & 745 & 0.13 & 6 \\
\hline $3 \mathrm{C} 132$ & $6.16 \pm 0.03$ & $-15.4 \pm 0.2$ & $50.11 \pm 0.37$ & 0.0164 & 378. & 54879 & 5.68 & 0.0 \\
\hline $3 \mathrm{C} 132$ & 2.21 & $-2.3 \pm 0.1$ & $3.15 \pm 0.28$ & $0.05 \pm 0.003$ & $37.05 \pm 8.88$ & 144 & 0.12 & 0 \\
\hline $3 \mathrm{C} 132$ & 11.81 & $2.0 \pm 0.0$ & $4.12 \pm 0.07$ & $0.34 \pm 0.003$ & $47.85 \pm 8.28$ & 151 & 1.29 & 2 \\
\hline 3C132 & $38.49 \pm 0.19$ & $5.3 \pm 0.0$ & $14.52 \pm 0.05$ & 0.0068 & 5703. & 4610 & 10.89 & 0.5 \\
\hline 3C132 & 12.35 & $8.0 \pm 0.0$ & $2.54 \pm 0.01$ & $1.33 \pm 0.008$ & $35.41 \pm 14.82$ & 91 & 2.32 & 3 \\
\hline 3C132 & $54.35 \pm 2.87$ & $8.4 \pm 0.0$ & $3.18 \pm 0.02$ & 0.0015 & 35172 . & 220 & 3.36 & 0.5 \\
\hline 3C132 & 23.31 & $13.1 \pm 0.0$ & $5.24 \pm 0.08$ & $0.24 \pm 0.002$ & $109.33 \pm 5.04$ & 328 & 2.66 & 1 \\
\hline $3 \mathrm{C} 133$ & 15.84 & $-29.8 \pm 0.1$ & $8.18 \pm 0.17$ & $0.05 \pm 0.002$ & $322.42 \pm 8.88$ & 1462 & 2.52 & 0 \\
\hline $3 \mathrm{C} 133$ & 14.58 & $-27.7 \pm 0.0$ & $3.28 \pm 0.11$ & $0.07 \pm 0.002$ & $38.56 \pm 8.28$ & 234 & 0.17 & 3 \\
\hline 3C133 & $10.16 \pm 0.08$ & $-16.8 \pm 0.3$ & $43.40 \pm 0.44$ & 0.0012 & 8199. & 41156 & 8.26 & 0.5 \\
\hline $3 \mathrm{C} 133$ & 3.20 & $-10.3 \pm 0.1$ & $5.98 \pm 0.38$ & $0.02 \pm 0.001$ & $219.63 \pm 14.82$ & 782 & 0.40 & 5 \\
\hline $3 \mathrm{C} 133$ & 0.77 & $-4.3 \pm 0.0$ & $1.49 \pm 0.05$ & $0.08 \pm 0.002$ & $4.89 \pm 5.04$ & 48 & 0.01 & 6 \\
\hline $3 \mathrm{C} 133$ & 5.98 & $-0.8 \pm 0.0$ & $3.96 \pm 0.07$ & $0.26 \pm 0.001$ & $12.37 \pm 6.75$ & 342 & 0.25 & 7 \\
\hline $3 \mathrm{C} 133$ & $67.68 \pm 0.54$ & $2.5 \pm 0.0$ & $10.40 \pm 0.05$ & 0.0009 & 73191. & 2362 & 13.71 & 0.0 \\
\hline $3 \mathrm{C} 133$ & 42.16 & $3.6 \pm 0.0$ & $3.10 \pm 0.02$ & $0.95 \pm 0.003$ & $47.99 \pm 6.26$ & 210 & 2.77 & 8 \\
\hline $3 \mathrm{C} 133$ & 48.15 & $7.6 \pm 0.0$ & $1.49 \pm 0.02$ & $1.01 \pm 0.019$ & $10.82 \pm 36.16$ & 48 & 0.32 & 2 \\
\hline $3 \mathrm{C} 133$ & 49.11 & $8.2 \pm 0.0$ & $2.84 \pm 0.02$ & $0.83 \pm 0.017$ & $69.32 \pm 6.19$ & 176 & 3.19 & 4 \\
\hline $3 \mathrm{C} 133$ & 11.35 & $11.9 \pm 1.1$ & $8.75 \pm 1.56$ & $0.02 \pm 0.001$ & $725.42 \pm 17.67$ & 1675 & 1.91 & 1 \\
\hline $4 \mathrm{C}+25.14$ & $1.86 \pm 0.06$ & $-23.5 \pm 1.1$ & $30.47 \pm 1.82$ & 0.0147 & 127. & 20291 & 0.95 & 0.5 \\
\hline $4 C+25.14$ & 0.14 & $-8.3 \pm 4.5$ & $16.30 \pm 5.51$ & $0.02 \pm 0.006$ & $12.84 \pm 8.88$ & 5802 & 0.09 & 2 \\
\hline $4 C+25.14$ & 1.13 & $-4.7 \pm 0.2$ & $4.59 \pm 0.88$ & $0.04 \pm 0.014$ & $21.83 \pm 8.28$ & 462 & 0.09 & 0 \\
\hline $4 C+25.14$ & 20.06 & $4.0 \pm 0.1$ & $1.82 \pm 0.18$ & $0.11 \pm 0.011$ & $41.54 \pm 14.82$ & 73 & 0.17 & 4 \\
\hline $4 C+25.14$ & 19.63 & $5.3 \pm 0.2$ & $7.15 \pm 0.38$ & $0.14 \pm 0.016$ & $130.63 \pm 5.04$ & 1117 & 2.61 & 3 \\
\hline $4 C+25.14$ & $8.95 \pm 0.11$ & $6.0 \pm 0.3$ & $27.53 \pm 0.40$ & 0.0022 & 4025 . & 16562 & 4.69 & 0.0 \\
\hline $4 C+25.14$ & 36.35 & $7.8 \pm 0.0$ & $2.10 \pm 0.03$ & $1.03 \pm 0.012$ & $41.83 \pm 6.75$ & 96 & 1.77 & 1 \\
\hline $4 C+25.14$ & 1.17 & $16.1 \pm 0.1$ & $1.76 \pm 0.13$ & $0.06 \pm 0.004$ & $15.94 \pm 6.26$ & 67 & 0.03 & 5 \\
\hline
\end{tabular}


Atomic gas around Perseus

\begin{tabular}{|c|c|c|c|c|c|c|c|c|}
\hline Source & $\begin{array}{l}\mathrm{T}_{B} \\
(\mathrm{~K}) \\
(1)\end{array}$ & $\begin{array}{c}\mathrm{V}_{\mathrm{LSR}} \\
\left(\mathrm{km} \mathrm{s}^{-1}\right) \\
(2)\end{array}$ & $\begin{array}{c}\Delta \mathrm{V} \\
\left(\mathrm{km} \mathrm{s}^{-1}\right) \\
(3)\end{array}$ & (4) & $\begin{array}{l}\mathrm{T}_{s} \\
(\mathrm{~K}) \\
(5)\end{array}$ & $\begin{array}{c}\mathrm{T}_{\mathrm{k}, \max } \\
(\mathrm{K}) \\
(6)\end{array}$ & $\begin{array}{c}\mathrm{N}(\mathrm{HI})_{20} \\
\left(\mathrm{~cm}^{-2}\right) \\
(7)\end{array}$ & $\bar{F}$ or $O$ \\
\hline $4 \mathrm{C}+25.14$ & 0.90 & $20.9 \pm 0.2$ & $3.82 \pm 0.41$ & $0.03 \pm 0.003$ & $32.05 \pm 6.19$ & 318 & 0.07 & 6 \\
\hline $4 \mathrm{C}+26.12$ & 7.28 & $-7.0 \pm 0.0$ & $2.60 \pm 0.05$ & $0.16 \pm 0.003$ & $53.06 \pm 8.88$ & 5802 & 0.44 & 1 \\
\hline $4 \mathrm{C}+26.12$ & $1.45 \pm 0.04$ & $-6.1 \pm 0.6$ & $59.78 \pm 1.92$ & 0.0049 & 295. & 1117 & 0.95 & 1.0 \\
\hline $4 \mathrm{C}+26.12$ & $6.59 \pm 0.11$ & $-3.9 \pm 0.2$ & $7.30 \pm 0.26$ & 0.0032 & 2088. & 96 & 0.94 & 1.0 \\
\hline $4 \mathrm{C}+26.12$ & $4.61 \pm 0.29$ & $1.7 \pm 0.1$ & $4.46 \pm 0.24$ & 0.0008 & 6111. & 67 & 0.40 & 0.0 \\
\hline $4 \mathrm{C}+26.12$ & 28.22 & $6.6 \pm 0.1$ & $8.95 \pm 0.20$ & $0.11 \pm 0.003$ & $182.48 \pm 14.82$ & 73 & 3.50 & 0 \\
\hline $4 \mathrm{C}+26.12$ & 28.39 & $6.7 \pm 0.0$ & $1.83 \pm 0.04$ & $0.30 \pm 0.005$ & $53.04 \pm 8.28$ & 462 & 0.56 & 2 \\
\hline $4 \mathrm{C}+27.07$ & $2.09 \pm 0.02$ & $-23.0 \pm 0.0$ & $24.44 \pm 0.27$ & 0.0124 & 169. & 73 & 0.86 & 1.0 \\
\hline $4 \mathrm{C}+27.07$ & 8.35 & $-5.2 \pm 0.1$ & $3.67 \pm 0.16$ & $0.10 \pm 0.003$ & $87.61 \pm 8.88$ & 5802 & 0.60 & 0 \\
\hline $4 \mathrm{C}+27.07$ & $16.85 \pm 0.14$ & $-1.7 \pm 0.0$ & $10.73 \pm 0.05$ & 0.0224 & 761. & 96 & 3.52 & 0.0 \\
\hline $4 \mathrm{C}+27.07$ & 15.70 & $-0.3 \pm 0.0$ & $2.96 \pm 0.05$ & $0.27 \pm 0.004$ & $61.24 \pm 8.28$ & 462 & 0.94 & 1 \\
\hline $4 \mathrm{C}+27.07$ & $2.24 \pm 0.12$ & $3.1 \pm 0.3$ & $20.01 \pm 0.32$ & 0.0023 & 971. & 1117 & 0.79 & 0.0 \\
\hline $4 \mathrm{C}+27.14$ & -1.05 & $-41.5 \pm 0.1$ & $1.74 \pm 0.18$ & $0.08 \pm 0.007$ & $3.58 \pm 8.88$ & 66 & 0.01 & 4 \\
\hline $4 \mathrm{C}+27.14$ & 13.09 & $-35.2 \pm 0.0$ & $2.49 \pm 0.11$ & $0.29 \pm 0.012$ & $34.00 \pm 8.28$ & 135 & 0.47 & 5 \\
\hline $4 \mathrm{C}+27.14$ & $14.70 \pm 0.24$ & $-34.1 \pm 0.1$ & $20.31 \pm 0.25$ & 0.0172 & 864. & 9019 & 5.80 & 0.0 \\
\hline $4 \mathrm{C}+27.14$ & 8.28 & $-31.2 \pm 0.3$ & $7.65 \pm 0.43$ & $0.14 \pm 0.005$ & $65.97 \pm 5.04$ & 1278 & 1.33 & 7 \\
\hline $4 \mathrm{C}+27.14$ & 6.28 & $-28.9 \pm 0.0$ & $1.19 \pm 0.07$ & $0.23 \pm 0.011$ & $13.99 \pm 14.82$ & 30 & 0.07 & 6 \\
\hline $4 \mathrm{C}+27.14$ & 3.20 & $-20.7 \pm 0.2$ & $3.89 \pm 0.42$ & $0.05 \pm 0.004$ & $85.24 \pm 6.75$ & 330 & 0.32 & 8 \\
\hline $4 \mathrm{C}+27.14$ & $12.75 \pm 0.41$ & $-12.9 \pm 0.1$ & $8.42 \pm 0.27$ & 0.0052 & 2478 . & 1548 & 2.09 & 0.0 \\
\hline $4 \mathrm{C}+27.14$ & 34.04 & $-0.1 \pm 0.1$ & $2.84 \pm 0.31$ & $0.14 \pm 0.016$ & $45.07 \pm 6.26$ & 176 & 0.35 & 1 \\
\hline $4 \mathrm{C}+27.14$ & 33.23 & $2.2 \pm 0.1$ & $12.26 \pm 0.46$ & $0.21 \pm 0.020$ & $161.68 \pm 36.16$ & 3287 & 8.06 & 0 \\
\hline $4 \mathrm{C}+27.14$ & $19.03 \pm 1.55$ & $2.8 \pm 0.3$ & $19.60 \pm 0.50$ & 0.0145 & 1320 . & 8396 & 7.23 & 0.0 \\
\hline $4 \mathrm{C}+27.14$ & 28.87 & $4.2 \pm 0.3$ & $2.86 \pm 0.74$ & $0.10 \pm 0.019$ & $13.99 \pm 6.19$ & 178 & 0.07 & 3 \\
\hline $4 \mathrm{C}+27.14$ & $38.30 \pm 2.39$ & $5.0 \pm 0.0$ & $5.66 \pm 0.14$ & 0.0044 & 8820. & 700 & 4.23 & 0.0 \\
\hline $4 \mathrm{C}+27.14$ & 40.88 & $6.9 \pm 0.0$ & $2.18 \pm 0.05$ & $1.10 \pm 0.022$ & $12.82 \pm 17.67$ & 103 & 0.60 & 2 \\
\hline $4 \mathrm{C}+28.06$ & $2.07 \pm 0.05$ & $-6.9 \pm 0.3$ & $39.13 \pm 0.60$ & 0.0021 & 984. & 330 & 1.23 & 1.0 \\
\hline $4 \mathrm{C}+28.06$ & 4.83 & $-6.8 \pm 0.1$ & $4.80 \pm 0.17$ & $0.07 \pm 0.002$ & $73.35 \pm 8.88$ & 66 & 0.51 & 2 \\
\hline $4 \mathrm{C}+28.06$ & $16.89 \pm 0.12$ & $0.3 \pm 0.0$ & $11.72 \pm 0.11$ & 0.0058 & 2937. & 176 & 3.85 & 1.0 \\
\hline $4 \mathrm{C}+28.06$ & 14.32 & $1.5 \pm 0.0$ & $5.31 \pm 0.07$ & $0.28 \pm 0.004$ & $69.60 \pm 8.28$ & 135 & 2.02 & 3 \\
\hline $4 \mathrm{C}+28.06$ & 12.98 & $3.3 \pm 0.0$ & $1.85 \pm 0.06$ & $0.24 \pm 0.007$ & $27.27 \pm 14.82$ & 30 & 0.24 & 1 \\
\hline $4 \mathrm{C}+28.06$ & 1.16 & $7.8 \pm 0.1$ & $1.70 \pm 0.23$ & $0.03 \pm 0.003$ & $36.98 \pm 5.04$ & 1278 & 0.04 & 0 \\
\hline $4 \mathrm{C}+28.07$ & $0.73 \pm 0.02$ & $-16.5 \pm 0.7$ & $56.11 \pm 1.46$ & 0.0034 & 212. & 178 & 0.35 & 0.0 \\
\hline $4 \mathrm{C}+28.07$ & 20.10 & $-6.3 \pm 0.0$ & $2.36 \pm 0.10$ & $0.15 \pm 0.009$ & $58.15 \pm 8.28$ & 135 & 0.40 & 1 \\
\hline $4 \mathrm{C}+28.07$ & 19.34 & $-5.7 \pm 0.1$ & $6.19 \pm 0.42$ & $0.08 \pm 0.009$ & $169.27 \pm 8.88$ & 66 & 1.71 & 2 \\
\hline $4 \mathrm{C}+28.07$ & 11.90 & $0.7 \pm 0.1$ & $3.02 \pm 0.08$ & $0.57 \pm 0.019$ & $16.40 \pm 14.82$ & 30 & 0.55 & 4 \\
\hline $4 \mathrm{C}+28.07$ & $9.04 \pm 0.28$ & $1.2 \pm 0.1$ & $16.08 \pm 0.13$ & 0.0019 & 4685. & 176 & 2.79 & 0.0 \\
\hline $4 \mathrm{C}+28.07$ & $31.30 \pm 0.71$ & $1.4 \pm 0.0$ & $4.65 \pm 0.02$ & 0.0045 & 6920 . & 3287 & 2.83 & 0.0 \\
\hline $4 \mathrm{C}+28.07$ & 6.65 & $3.2 \pm 0.2$ & $2.94 \pm 0.25$ & $0.19 \pm 0.026$ & $39.45 \pm 5.04$ & 1278 & 0.42 & 0 \\
\hline $4 \mathrm{C}+28.07$ & 0.99 & $6.7 \pm 1.4$ & $6.25 \pm 1.93$ & $0.02 \pm 0.003$ & $139.49 \pm 6.75$ & 330 & 0.28 & 3 \\
\hline $4 \mathrm{C}+29.05$ & $1.57 \pm 0.03$ & $-11.4 \pm 0.2$ & $43.60 \pm 0.47$ & 0.0160 & 99 . & 330 & 0.96 & 1.0 \\
\hline $4 \mathrm{C}+29.05$ & 0.67 & $-8.0 \pm 0.1$ & $2.20 \pm 0.31$ & $0.03 \pm 0.003$ & $32.58 \pm 8.88$ & 66 & 0.04 & 0 \\
\hline $4 \mathrm{C}+29.05$ & $6.13 \pm 0.08$ & $-4.4 \pm 0.1$ & $16.62 \pm 0.13$ & 0.0082 & 752. & 1278 & 1.97 & 1.0 \\
\hline $4 \mathrm{C}+29.05$ & 6.28 & $-1.3 \pm 0.0$ & $3.93 \pm 0.08$ & $0.14 \pm 0.003$ & $49.20 \pm 8.28$ & 135 & 0.54 & 1 \\
\hline $4 \mathrm{C}+29.05$ & $7.87 \pm 0.10$ & $-0.4 \pm 0.0$ & $7.94 \pm 0.07$ & 0.0017 & 4770 . & 30 & 1.22 & 0.0 \\
\hline $4 \mathrm{C}+30.04$ & $1.30 \pm 0.04$ & $-4.8 \pm 0.7$ & $42.10 \pm 1.04$ & 0.0191 & 68. & 38738 & 0.74 & 1.0 \\
\hline $4 \mathrm{C}+30.04$ & 23.10 & $-2.9 \pm 0.0$ & $2.62 \pm 0.02$ & $0.67 \pm 0.007$ & $45.83 \pm 8.88$ & 149 & 1.57 & 1 \\
\hline $4 \mathrm{C}+30.04$ & 19.23 & $1.0 \pm 0.0$ & $1.83 \pm 0.05$ & $0.51 \pm 0.013$ & $40.71 \pm 8.28$ & 73 & 0.74 & 3 \\
\hline $4 \mathrm{C}+30.04$ & $35.95 \pm 0.22$ & $2.3 \pm 0.0$ & $10.46 \pm 0.03$ & 0.0109 & 3326. & 2391 & 7.33 & 0.0 \\
\hline $4 \mathrm{C}+30.04$ & 7.00 & $4.1 \pm 0.1$ & $4.61 \pm 0.12$ & $0.43 \pm 0.004$ & $15.64 \pm 14.82$ & 465 & 0.60 & 2 \\
\hline $4 \mathrm{C}+30.04$ & 0.09 & $10.4 \pm 0.1$ & $3.16 \pm 0.15$ & $0.10 \pm 0.004$ & $1.99 \pm 5.04$ & 217 & 0.01 & 0 \\
\hline $4 \mathrm{C}+30.04$ & $4.26 \pm 0.11$ & $13.7 \pm 0.3$ & $13.23 \pm 0.50$ & 0.0161 & 267. & 3822 & 1.06 & 0.0 \\
\hline $4 \mathrm{C}+33.10$ & 4.68 & $-52.8 \pm 0.1$ & $2.36 \pm 0.13$ & $0.12 \pm 0.006$ & $33.86 \pm 8.88$ & 149 & 0.19 & 4 \\
\hline $4 \mathrm{C}+33.10$ & $12.70 \pm 0.39$ & $-46.2 \pm 0.2$ & $17.41 \pm 0.43$ & 0.0037 & 3394. & 1017 & 4.29 & 0.0 \\
\hline
\end{tabular}




\begin{tabular}{|c|c|c|c|c|c|c|c|c|}
\hline Source & $\begin{array}{l}\mathrm{T}_{B} \\
(\mathrm{~K}) \\
(1)\end{array}$ & $\begin{array}{c}\mathrm{V}_{\mathrm{LSR}} \\
\left(\mathrm{km} \mathrm{s}^{-1}\right) \\
(2)\end{array}$ & $\begin{array}{c}\Delta \mathrm{V} \\
\left(\mathrm{km} \mathrm{s}^{-1}\right) \\
(3)\end{array}$ & (4) & $\begin{array}{l}\mathrm{T}_{s} \\
(\mathrm{~K}) \\
(5)\end{array}$ & $\begin{array}{c}\mathrm{T}_{\mathrm{k}, \max } \\
(\mathrm{K}) \\
(6)\end{array}$ & $\begin{array}{c}\mathrm{N}(\mathrm{HI})_{20} \\
\left(\mathrm{~cm}^{-2}\right) \\
(7)\end{array}$ & $\begin{array}{c}F \text { or } O \\
(8)\end{array}$ \\
\hline $4 \mathrm{C}+33.10$ & 10.10 & $-44.8 \pm 0.0$ & $3.06 \pm 0.09$ & $0.20 \pm 0.006$ & $52.19 \pm 8.28$ & 73 & 0.62 & 5 \\
\hline $4 C+33.10$ & $12.34 \pm 1.36$ & $-43.2 \pm 0.3$ & $4.50 \pm 0.29$ & 0.0039 & 3180. & 1017 & 1.08 & 0.0 \\
\hline $4 \mathrm{C}+33.10$ & $5.44 \pm 0.30$ & $-30.6 \pm 0.2$ & $6.82 \pm 0.54$ & 0.0026 & 2081. & 1017 & 0.72 & 0.0 \\
\hline $4 \mathrm{C}+33.10$ & 3.88 & $-16.1 \pm 0.1$ & $2.77 \pm 0.25$ & $0.07 \pm 0.005$ & $45.43 \pm 14.82$ & 465 & 0.17 & 6 \\
\hline $4 C+33.10$ & 4.41 & $-8.8 \pm 0.1$ & $4.27 \pm 0.17$ & $0.21 \pm 0.005$ & $19.31 \pm 5.04$ & 217 & 0.33 & 7 \\
\hline $4 C+33.10$ & $34.42 \pm 0.45$ & $-6.8 \pm 0.1$ & $24.91 \pm 0.23$ & 0.0118 & 2933. & 1017 & 16.62 & 0.0 \\
\hline $4 C+33.10$ & 23.35 & $-3.2 \pm 0.1$ & $3.57 \pm 0.12$ & $0.62 \pm 0.010$ & $48.13 \pm 6.75$ & 443 & 2.07 & 0 \\
\hline $4 C+33.10$ & 43.26 & $1.9 \pm 0.1$ & $4.21 \pm 0.21$ & $0.95 \pm 0.012$ & $69.69 \pm 6.26$ & 13556 & 5.43 & 1 \\
\hline $4 C+33.10$ & 42.58 & $5.8 \pm 0.1$ & $3.03 \pm 0.11$ & $0.83 \pm 0.028$ & $70.14 \pm 6.19$ & 684 & 3.44 & 2 \\
\hline $4 C+33.10$ & $26.72 \pm 1.09$ & $7.3 \pm 0.2$ & $5.59 \pm 0.17$ & 0.0116 & 2317. & 1017 & 2.91 & 0.0 \\
\hline $4 C+33.10$ & 7.53 & $9.7 \pm 0.1$ & $2.46 \pm 0.14$ & $0.19 \pm 0.007$ & $36.57 \pm 36.16$ & 6621 & 0.34 & 3 \\
\hline $4 \mathrm{C}+34.07$ & $2.00 \pm 0.00$ & $-22.7 \pm 0.0$ & $3.07 \pm 0.10$ & 0.0132 & 153. & 3403 & 0.12 & 1.0 \\
\hline $4 \mathrm{C}+34.07$ & $70 \pm 0.03$ & $-9.1 \pm 0.3$ & $46.64 \pm 0.63$ & 0.0002 & 10602. & 217 & 1.14 & 0.0 \\
\hline $4 \mathrm{C}+34.07$ & 20.20 & $-2.2 \pm 0.0$ & $1.84 \pm 0.05$ & $0.24 \pm 0.007$ & $21.05 \pm 8.88$ & 149 & 0.18 & 0 \\
\hline $4 \mathrm{C}+34.07$ & 20.62 & $-1.0 \pm 0.0$ & $5.63 \pm 0.10$ & $0.15 \pm 0.009$ & $140.20 \pm 8.28$ & 73 & 2.26 & 2 \\
\hline $4 C+34.07$ & $21 \pm 0.09$ & $0.3 \pm 0.0$ & $12.48 \pm 0.11$ & 0.0086 & 845. & 47549 & 1.75 & 0.0 \\
\hline $4 C+34.07$ & 18.91 & $1.1 \pm 0.0$ & $1.79 \pm 0.11$ & $0.09 \pm 0.006$ & $63.79 \pm 14.82$ & 465 & 0.21 & 1 \\
\hline $4 \mathrm{C}+34.09$ & $2.30 \pm 0.05$ & $-10.2 \pm 0.5$ & $61.68 \pm 1.46$ & 0.0051 & 452. & 217 & 2.18 & 0.0 \\
\hline $4 C+34.09$ & 0.74 & $-8.0 \pm 0.0$ & $1.69 \pm 0.03$ & $0.22 \pm 0.004$ & $4.79 \pm 8.88$ & 149 & 0.03 & 2 \\
\hline $4 \mathrm{C}+34.09$ & $7.65 \pm 0.12$ & $-2.1 \pm 0.1$ & $8.36 \pm 0.20$ & 0.0017 & 4607. & 83144 & 1.25 & 1.0 \\
\hline $4 C+34.09$ & 35.09 & $1.1 \pm 0.0$ & $2.70 \pm 0.06$ & $0.24 \pm 0.005$ & $93.71 \pm 8.28$ & 73 & 1.19 & 0 \\
\hline $4 C+34.09$ & 27.38 & $4.7 \pm 0.1$ & $9.80 \pm 0.11$ & $0.19 \pm 0.002$ & $157.27 \pm 14.82$ & 465 & 5.79 & 1 \\
\hline $5 \mathrm{C} 06.237$ & $2.30 \pm 0.05$ & $-7.4 \pm 0.3$ & $40.50 \pm 0.71$ & 0.0031 & 750 . & 217 & 1.48 & 0.0 \\
\hline $5 \mathrm{C} 06.237$ & 14.03 & $-1.3 \pm 0.0$ & $3.42 \pm 0.07$ & $0.40 \pm 0.004$ & $52.28 \pm 8.88$ & 149 & 1.40 & 1 \\
\hline $5 \mathrm{C} 06.237$ & $14.48 \pm 0.20$ & $-0.5 \pm 0.0$ & $9.96 \pm 0.10$ & 0.0094 & 1545. & 465 & 2.81 & 1.0 \\
\hline 5C06.237 & 6.37 & $2.0 \pm 0.1$ & $2.18 \pm 0.16$ & $0.10 \pm 0.006$ & $62.11 \pm 8.28$ & 73 & 0.27 & 0 \\
\hline B20218+35 & $5.23 \pm 0.03$ & $-57.9 \pm 0.0$ & $14.08 \pm 0.10$ & 0.0112 & 471. & 217 & 1.35 & 0.0 \\
\hline B20218+35 & $2.25 \pm 0.03$ & $-10.4 \pm 0.2$ & $45.22 \pm 0.53$ & 0.0026 & 881. & 4332 & 1.54 & 0.0 \\
\hline B20218+35 & 3.89 & $-4.5 \pm 0.2$ & $2.77 \pm 0.50$ & $0.03 \pm 0.011$ & $116.48 \pm 8.88$ & 149 & 0.19 & 0 \\
\hline B20218+35 & $12.27 \pm 0.36$ & $-0.3 \pm 0.0$ & $10.40 \pm 0.12$ & 0.0028 & 4331. & 44682 & 2.48 & 0.0 \\
\hline B20218+35 & 6.84 & $-0.3 \pm 0.9$ & $6.24 \pm 2.03$ & $0.04 \pm 0.030$ & $49.87 \pm 14.82$ & 465 & 0.26 & 1 \\
\hline B20218+35 & 8.44 & $0.7 \pm 0.1$ & $2.82 \pm 0.34$ & $0.11 \pm 0.031$ & $62.21 \pm 8.28$ & 73 & 0.38 & 2 \\
\hline B20326+27 & $1.33 \pm 0.03$ & $-12.7 \pm 0.6$ & $64.39 \pm 1.51$ & 0.0037 & 364. & 217 & 0.96 & 1.0 \\
\hline B20326+27 & 19.44 & $0.4 \pm 0.0$ & $4.67 \pm 0.07$ & $0.30 \pm 0.003$ & $78.55 \pm 8.88$ & 14 & 2.12 & 0 \\
\hline B20326+27 & $19.93 \pm 0.14$ & $3.0 \pm 0.0$ & $13.20 \pm 0.05$ & 0.0184 & 1093. & 465 & 5.12 & 0.5 \\
\hline B20326+27 & 23.00 & $6.7 \pm 0.0$ & $4.56 \pm 0.06$ & $0.34 \pm 0.003$ & $83.42 \pm 8.28$ & 73 & 2.49 & 1 \\
\hline B20400+25 & $1.97 \pm 0.10$ & $-48.0 \pm 0.1$ & $5.04 \pm 0.32$ & 0.0017 & 1189. & 35507 & 0.18 & 0.0 \\
\hline B20400+25 & $1.91 \pm 0.04$ & $-27.4 \pm 0.5$ & $40.31 \pm 1.29$ & 0.0112 & 171. & 217 & 1.38 & 1.0 \\
\hline B20400+25 & 17.65 & $5.0 \pm 0.4$ & $14.63 \pm 0.63$ & $0.03 \pm 0.002$ & $607.24 \pm 8.28$ & 73 & 4.58 & 0 \\
\hline $00+25$ & 33.18 & $7.2 \pm 0.0$ & $2.17 \pm 0.04$ & $0.31 \pm 0.007$ & $67.41 \pm 14.82$ & 46 & 0.88 & 2 \\
\hline B20400+25 & 23.82 & $8.6 \pm 0.1$ & $4.79 \pm 0.10$ & $0.16 \pm 0.006$ & $54.35 \pm 8.88$ & 149 & 0.80 & 1 \\
\hline B20400+25 & $1.38 \pm 0.06$ & $19.9 \pm 0.3$ & $13.70 \pm 0.88$ & 0.0033 & 422 . & 555 & 0.30 & 0.0 \\
\hline B20411+34 & $2.34 \pm 0.27$ & $-28.4 \pm 4.6$ & $48.16 \pm 5.23$ & 0.0136 & 174. & 50696 & 1.90 & 0.0 \\
\hline B20411+34 & 1.44 & $-14.4 \pm 0.3$ & $3.86 \pm 0.66$ & $02 \pm 0.004$ & $14.83 \pm 8.88$ & 325 & 0.03 & 3 \\
\hline B20411+34 & $11.85 \pm 0.59$ & $-4.8 \pm 0.2$ & $27.39 \pm 0.65$ & 0.0133 & 899. & 16401 & 6.23 & 0.0 \\
\hline B20411+34 & 3.88 & $-3.3 \pm 0.2$ & $5.27 \pm 0.35$ & $0.07 \pm 0.003$ & $106.09 \pm 8.28$ & 607 & 0.74 & 2 \\
\hline B20411+34 & $18.58 \pm 0.70$ & $4.1 \pm 0.2$ & $8.50 \pm 0.26$ & 0.0080 & 2322. & 1577 & 3.07 & 0.0 \\
\hline B20411+34 & 35.65 & $5.1 \pm 0.1$ & $4.73 \pm 0.20$ & $0.90 \pm 0.128$ & $39.17 \pm 14.82$ & 489 & 3.27 & 1 \\
\hline B20411+34 & 25.16 & $8.5 \pm 1.5$ & $6.18 \pm 1.27$ & $0.18 \pm 0.073$ & $68.77 \pm 5.04$ & 835 & 1.53 & 0 \\
\hline NV0157+28 & $2.80 \pm 0.02$ & $-13.6 \pm 0.1$ & $40.62 \pm 0.25$ & 0.0083 & 340. & 446 & 1.91 & 0.0 \\
\hline NV0157+28 & $2.40 \pm 0.04$ & $-10.4 \pm 0.0$ & $4.52 \pm 0.09$ & 0.0022 & 1098. & 489 & 0.21 & 0.0 \\
\hline NV0157+28 & 14.89 & $-2.7 \pm 0.1$ & $9.76 \pm 0.18$ & $0.05 \pm 0.001$ & $302.10 \pm 8.88$ & 325 & 2.90 & 1 \\
\hline NV0157+28 & 13.69 & $-0.5 \pm 0.0$ & $1.96 \pm 0.12$ & $0.04 \pm 0.002$ & $25.80 \pm 8.28$ & 607 & 0.04 & 0 \\
\hline
\end{tabular}




\begin{tabular}{lcccccccc}
\hline \hline Source & $\begin{array}{c}\mathrm{T}_{B} \\
(\mathrm{~K})\end{array}$ & $\begin{array}{c}\mathrm{V}_{\mathrm{LSR}} \\
\left(\mathrm{km} \mathrm{s}^{-1}\right) \\
(1)\end{array}$ & $\begin{array}{c}\Delta \mathrm{V} \\
\left(\mathrm{km} \mathrm{s}^{-1}\right)\end{array}$ & $(3)$ & $(4)$ & $\begin{array}{c}\mathrm{T}_{s} \\
(\mathrm{~K})\end{array}$ & $\begin{array}{c}\mathrm{T}_{\mathrm{k}, \max } \\
(\mathrm{K})\end{array}$ & $\begin{array}{c}\mathrm{N}(\mathrm{HI})_{20} \\
\left(\mathrm{~cm}^{-2}\right)\end{array}$ \\
& $\begin{array}{l}(5) \\
(7)\end{array}$ & $(8)$ & $(8)$ \\
\hline NV0157+28 & $3.80 \pm 0.10$ & $2.7 \pm 0.2$ & $10.56 \pm 0.26$ & 0.0041 & 932. & 835 & 0.77 & 1.0 \\
\hline NV0232+34 & $2.69 \pm 0.03$ & $-6.5 \pm 0.1$ & $39.32 \pm 0.37$ & 0.0110 & 245. & 33787 & 1.68 & 0.0 \\
NV0232+34 & 20.16 & $-1.9 \pm 0.1$ & $2.04 \pm 0.16$ & $0.13 \pm 0.010$ & $43.04 \pm 8.28$ & 607 & 0.22 & 0 \\
NV0232+34 & 22.52 & $-0.1 \pm 0.2$ & $8.49 \pm 0.71$ & $0.06 \pm 0.014$ & $321.07 \pm 8.88$ & 325 & 3.10 & 2 \\
NV0232+34 & 22.90 & $0.4 \pm 0.0$ & $1.82 \pm 0.09$ & $0.20 \pm 0.012$ & $37.38 \pm 14.82$ & 489 & 0.27 & 3 \\
NV0232+34 & 14.61 & $3.0 \pm 0.1$ & $2.11 \pm 0.19$ & $0.09 \pm 0.008$ & $30.54 \pm 5.04$ & 835 & 0.11 & 1 \\
\hline \hline
\end{tabular}

